\title{
EIGENVALUES OF TOEPLITZ MINIMAL SYSTEMS OF FINITE TOPOLOGICAL RANK
}

\author{
FABIEN DURAND, ALEXANDER FRANK, AND ALEJANDRO MAASS
}

\begin{abstract}
In this article we characterize measure theoretical eigenvalues of Toeplitz Bratteli-Vershik minimal systems of finite topological rank which are not associated to a continuous eigenfunction. Several examples are provided to illustrate the different situations that can occur.
\end{abstract}

\section{INTRODUCTION}

Seminal results by M. Dekking Dek78 and B. Host Hos86 state that eigenvalues of primitive substitution dynamical systems are always associated to continuous eigenfunctions. Thus the topological and measure theoretical Kronecker factors coincide. It is natural to ask whether this phenomenon is still true for other classes of minimal Cantor systems. Most of the answers we have are negative.

Substitution dynamical systems correspond to expansive minimal Cantor systems having a periodic or stationary Bratteli-Vershik representation [DHS99. A natural class to explore extending the former one are linearly recurrent minimal Cantor systems, which correspond to those systems having a Bratteli-Vershik representation with a bounded number of incidence matrices. In CDHM03 and BDM05] necessary and sufficient conditions based only on the combinatorial structure of the Bratteli diagrams are given for this class of systems, allowing to differentiate continuous and measure theoretical but non continuous eigenvalues. The more general class of topological finite rank minimal Cantor systems is explored in BDM10, providing new examples and conditions to differentiate the topological and measure theoretical Kronecker factors.

It is known that any countable subgroup of the torus $\mathbb{S}^{1}=\{z \in \mathbb{C} ;|z|=1\}$ containing infinitely many rationals can be the set of eigenvalues of a Toeplitz system Iwa96, DL96. Nevertheless, in the class of finite rank systems, Toeplitz systems exhibit a completely different behavior. Indeed, if a Toeplitz system is linearly recurrent then all its eigenvalues are associated to continuous eigenfunctions and if it has finite topological rank just a few extra non continuous eigenvalues can appear and they are rational BDM10. So the assumption of finite topological

Date: March 16, 2014.

1991 Mathematics Subject Classification. Primary: 54H20; Secondary: 37B20.

Key words and phrases. Toeplitz systems, finite rank Bratteli-Vershik dynamical systems, eigenvalues.

This research was partially supported by grants Basal-CMM \& Fondap 15090007, Proyecto Anillo ACT1103, ANR Subtile and the cooperation project Mathamsud DYSTIL. The third author thanks Chaire Bézout at University of Paris Est and U. Picardie Jules Verne where this research was finished.

We are very grateful to the anonymous referee who helped significantly to improve the presentation of this article. 
rank restricts the possibilities of non continuous eigenvalues to some particular ones. The purpose of this work is to study the nature of these particular non continuous eigenvalues of finite rank Toeplitz systems.

Our main result (Theorem 3) states a necessary and sufficient condition for $\lambda=$ $\exp (2 i \pi a / b)$, where $a, b$ are integers with $(a, b)=1$, to be a non continuous eigenvalue of a finite topological rank Toeplitz system. This condition shows that non continuous eigenvalues are very rare and impose particular local orders to the associated Bratteli-Vershik representations. In addition, even if this condition looks abstract, it is easily computable and allows to produce concrete examples, showing particular behaviors of the group of eigenvalues in relation to the set of ergodic measures.

The article is organized as follows. Section 2 contains the main definitions concerning eigenvalues of dynamical systems and Bratteli-Vershik representations, in particular the concept of Toeplitz minimal Cantor system of finite topological rank. In Section 3 we give the main result of the article and its corollaries. In particular, we exhibit a relation between the number of ergodic measures and the number of non continuous eigenvalues in the class of Toeplitz minimal Cantor systems of finite topological rank. Main technical lemmas used in the proofs are given in Section 4 and the proofs of the main result and its corollaries in Section 5. Finally, in Section 6 we provide several examples to illustrate the main result, its consequences and the fact that our condition is computable.

\section{BASIC DEFINITIONS}

2.1. Dynamical systems and eigenvalues. A topological dynamical system, or just dynamical system, is a compact Hausdorff space $X$ together with a homeomorphism $T: X \rightarrow X$. We use the notation $(X, T)$. If $X$ is a Cantor set (i.e., $X$ has a countable basis of closed and open sets and it has no isolated points) we say that the system is Cantor. A dynamical system is minimal if all orbits are dense in $X$, or equivalently the only non empty closed invariant set is $X$.

A complex number $\lambda$ is a continuous eigenvalue of $(X, T)$ if there exists a continuous function $f: X \rightarrow \mathbb{C}, f \neq 0$, such that $f \circ T=\lambda f ; f$ is called a continuous eigenfunction (associated to $\lambda$ ). Let $\mu$ be a $T$-invariant probability measure, i.e., $T \mu=\mu$, defined on the Borel $\sigma$-algebra of $X$. A complex number $\lambda$ is an eigenvalue of the dynamical system $(X, T)$ with respect to $\mu$ if there exists $f \in L^{2}(X, \mu)$, $f \neq 0$, such that $f \circ T=\lambda f ; f$ is called an eigenfunction (associated to $\lambda$ ). If $\mu$ is ergodic, then every eigenvalue has modulus 1 and every eigenfunction has a constant modulus $\mu$-almost surely. Of course, continuous eigenvalues are eigenvalues.

2.2. Bratteli-Vershik representations. Let $(X, T)$ be a minimal Cantor system. It can be represented by an ordered Bratteli diagram together with the Vershik transformation acting on it. For details on this theory see HPS92 or Dur10. This couple is called a Bratteli-Vershik representation of the system. We give a brief outline of this construction emphasizing the notations in this paper.

2.2.1. Bratteli diagrams. A Bratteli diagram is an infinite graph $(V, E)$ which consists of a vertex set $V$ and an edge set $E$, both of which are divided into levels $V=V_{0} \cup V_{1} \cup \ldots, E=E_{1} \cup E_{2} \cup \ldots$ and all levels are pairwise disjoint. The set $V_{0}$ is a singleton $\left\{v_{0}\right\}$ and for all $n \geq 1$ edges in $E_{n}$ join vertices in $V_{n-1}$ to vertices 
in $V_{n}$. It is also required that every vertex in $V_{n}$ is the "end-point" of some edge in $E_{n}$ for $n \geq 1$ and an "initial-point" of some edge in $E_{n+1}$ for $n \geq 0$. We set $\# V_{n}=d_{n}$ for all $n \geq 1$.

Fix $n \geq 1$. We call level $n$ of the diagram to the subgraph consisting of the vertices in $V_{n-1} \cup V_{n}$ and the edges $E_{n}$ between these vertices. Level 1 is called the hat of the Bratteli diagram. We describe the edge set $E_{n}$ using a $V_{n-1} \times V_{n}$ incidence matrix $M_{n}$ for which its $\left(t_{1}, t_{2}\right)$ entry is the number of edges in $E_{n}$ joining vertex $t_{1} \in V_{n-1}$ with vertex $t_{2} \in V_{n}$. We also set $P_{n}=M_{2} \cdots M_{n}$ with the convention that $P_{1}=I$, where $I$ denotes the identity matrix. The number of paths joining $v_{0} \in V_{0}$ and a vertex $t \in V_{n}$ is given by coordinate $t$ of the height row vector $h_{n}=\left(h_{n}(t) ; t \in V_{n}\right) \in \mathbb{N}^{d_{n}}$. Notice that $h_{1}=M_{1}$ and $h_{n}=h_{1} P_{n}$.

We also consider several levels at the same time. For integers $0 \leq m<n$ we denote by $E_{m, n}$ the set of all paths in the graph joining vertices of $V_{m}$ with vertices of $V_{n}$. We define matrices $P_{m, n}=M_{m+1} \cdots M_{n}$ with the convention that $P_{n, n}=I$ for $1 \leq m \leq n$. Clearly, coordinate $P_{m, n}\left(t_{1}, t_{2}\right)$ of matrix $P_{m, n}$ is the number of paths in $E_{m, n}$ from vertex $t_{1} \in V_{m}$ to vertex $t_{2} \in V_{n}$. It can be verified that $h_{n}=h_{m} P_{m, n}$.

We need to notice that the incidence matrices defined above correspond to the transpose of the matrices defined at the classical reference in this theory [HPS92. This choice is done to simplify the understanding and reading of the article.

2.2.2. Ordered Bratteli diagrams and Bratteli-Vershik representations. An ordered Bratteli diagram is a triple $B=(V, E, \preceq)$, where $(V, E)$ is a Bratteli diagram and $\preceq$ is a partial ordering on $E$ such that: edges $e$ and $e^{\prime}$ are comparable if and only if they have the same end-point. This partial ordering naturally defines maximal and minimal edges and paths. Also, the partial ordering of $E$ induces another one on paths of $E_{m, n}$, where $0 \leq m<n:\left(e_{m+1}, \ldots, e_{n}\right) \preceq\left(f_{m+1}, \ldots, f_{n}\right)$ if and only if there is $m+1 \leq i \leq n$ such that $e_{j}=f_{j}$ for $i<j \leq n$ and $e_{i} \preceq f_{i}$.

Given a strictly increasing sequence of integers $\left(n_{k}\right)_{k \geq 0}$ with $n_{0}=0$ one defines the contraction or telescoping of $B=(V, E, \preceq)$ with respect to $\left(n_{k}\right)_{k \geq 0}$ as

$$
\left(\left(V_{n_{k}}\right)_{k \geq 0},\left(E_{n_{k}, n_{k+1}}\right)_{k \geq 0}, \preceq\right),
$$

where $\preceq$ is the order induced in each set of edges $E_{n_{k}, n_{k+1}}$. The converse operation is called microscoping (see [HPS92] for more details).

Given an ordered Bratteli diagram $B=(V, E, \preceq)$ one defines $X_{B}$ as the set of infinite paths $\left(x_{1}, x_{2}, \ldots\right)$ starting in $v_{0}$ such that for all $n \geq 1$ the end-point of $x_{n} \in E_{n}$ is the initial-point of $x_{n+1} \in E_{n+1}$. We topologize $X_{B}$ by postulating a basis of open sets, namely the family of cylinder sets

$$
\left[e_{1}, e_{2}, \ldots, e_{n}\right]=\left\{\left(x_{1}, x_{2}, \ldots\right) \in X_{B} ; x_{i}=e_{i}, \text { for } 1 \leq i \leq n\right\} .
$$

Each $\left[e_{1}, e_{2}, \ldots, e_{n}\right]$ is also closed, as is easily seen, and so $X_{B}$ is a compact, totally disconnected metrizable space.

When there is a unique $\left(x_{1}, x_{2}, \ldots\right) \in X_{B}$ such that $x_{n}$ is (locally) maximal for any $n \geq 1$ and a unique $\left(y_{1}, y_{2}, \ldots\right) \in X_{B}$ such that $y_{n}$ is (locally) minimal for any $n \geq 1$, one says that $B=(V, E, \preceq)$ is a properly ordered Bratteli diagram. Call these particular points $x_{\max }$ and $x_{\min }$ respectively. In this case one defines the dynamic $V_{B}$ over $X_{B}$ called the Vershik map. Let $x=\left(x_{1}, x_{2}, \ldots\right) \in X_{B} \backslash\left\{x_{\max }\right\}$ and let $n \geq 1$ be the smallest integer so that $x_{n}$ is not a maximal edge. Let $y_{n}$ 
be the successor of $x_{n}$ for the local order and $\left(y_{1}, \ldots, y_{n-1}\right)$ be the unique minimal path in $E_{0, n-1}$ connecting $v_{0}$ with the initial vertex of $y_{n}$. One sets $V_{B}(x)=$ $\left(y_{1}, \ldots, y_{n-1}, y_{n}, x_{n+1}, \ldots\right)$ and $V_{B}\left(x_{\max }\right)=x_{\min }$.

The dynamical system $\left(X_{B}, V_{B}\right)$ is minimal. It is called the Bratteli-Vershik system generated by $B=(V, E, \preceq)$. The dynamical system induced by any telescoping of $B$ is topologically conjugate to $\left(X_{B}, V_{B}\right)$. In HPS92] it is proved that any minimal Cantor system $(X, T)$ is topologically conjugate to a Bratteli-Vershik system $\left(X_{B}, V_{B}\right)$. One says that $\left(X_{B}, V_{B}\right)$ is a Bratteli-Vershik representation of $(X, T)$. In what follows we identify $(X, T)$ with any of its Bratteli-Vershik representations.

2.2.3. Minimal Cantor systems of finite topological rank. A minimal Cantor system is of finite (topological) rank if it admits a Bratteli-Vershik representation such that the number of vertices per level is uniformly bounded by some integer $d$. The minimum possible value of $d$ is called the topological rank of the system. We observe that topological and measure theoretical finite rank notions are completely different. For instance, systems of topological rank one correspond to odometers, whereas in the measure theoretical sense there are rank one systems that are expansive as classical Chacon's example.

To have a better understanding of the dynamics of a minimal Cantor system, and in particular to understand its group of eigenvalues, one needs to work with a "good" Bratteli-Vershik representation. In the context of minimal Cantor systems of finite rank $d$ we will consider representations verifying:

(H1) The entries of $h_{1}$ are all equal to 1.

(H2) For every $n \geq 2, M_{n}>0$.

(H3) For every $n \geq 2, d_{n}$ is equal to $d$.

(H4) For every $n \geq 2$, all maximal edges of $E_{n}$ start in the same vertex of $V_{n-1}$.

A Bratteli-Vershik representation of a minimal Cantor system $(X, T)$ verifying $(\mathrm{H} 1)$, (H2), (H3) and (H4) will be called proper. In this case, to simplify notations and avoid the excessive use of indexes, we will identify $V_{n}$ with $\{1, \ldots, d\}$ for all $n \geq 1$. The level $n$ will be clear from the context.

It is not difficult to prove that a minimal Cantor system of finite rank $d$ has a proper representation. We give a brief outline for completeness. We start from a given Bratteli-Vershik representation that we transform by telescoping. Condition (H1) follows by splitting the first level to separate all arrows in the hat and then duplicating accordingly the arrows of the second level. By minimality there is a telescoping of the diagram such that (H2) holds HPS92. Another telescoping to the levels where $\# V_{n}=d$ produces (H3). Property (H4) follows from a compactness argument and a series of telescopings: if this is not possible, then we can construct two disjoint maximal points and we get a contradiction.

A minimal Cantor system is linearly recurrent if it admits a proper Bratteli-Vershik representation such that the set $\left\{M_{n} ; n \geq 1\right\}$ is finite. Clearly, linearly recurrent minimal Cantor systems are of finite rank (see DHS99, Dur00, Dur03] and CDHM03 for more details on this class of systems).

2.2.4. Associated Kakutani-Rohlin partitions. Let $B=(V, E, \preceq)$ be a properly ordered Bratteli diagram and $(X, T)$ the associated minimal Cantor system. This diagram defines for each $n \geq 0$ a clopen Kakutani-Rohlin partition of $X$ : for $n=0$, 
$\mathcal{P}_{0}=\left\{B_{0}\left(v_{0}\right)\right\}$, where $B_{0}\left(v_{0}\right)=X$, and for $n \geq 1$

$$
\mathcal{P}_{n}=\left\{T^{-j} B_{n}(t) ; t \in V_{n}, 0 \leq j<h_{n}(t)\right\},
$$

where $B_{n}(t)=\left[e_{1}, \ldots, e_{n}\right]$ and $\left(e_{1}, \ldots, e_{n}\right)$ is the unique maximal path from $v_{0}$ to vertex $t \in V_{n}$. For each $t \in V_{n}$ the set $\left\{T^{-j} B_{n}(t) ; 0 \leq j<h_{n}(t)\right\}$ is called the tower $t$ of $\mathcal{P}_{n}$. It corresponds to the set of all paths from $v_{0}$ to $t \in V_{n}$ (there are exactly $h_{n}(t)$ of such paths). Denote by $\mathcal{T}_{n}$ the $\sigma$-algebra generated by the partition $\mathcal{P}_{n}$. The map $\tau_{n}: X \rightarrow V_{n}$ is given by $\tau_{n}(x)=t$ if $x$ belongs to tower $t$ of $\mathcal{P}_{n}$. The entrance time of $x$ to $B_{n}\left(\tau_{n}(x)\right)$ is given by $r_{n}(x)=\min \left\{j \geq 0 ; T^{j} x \in B_{n}\left(\tau_{n}(x)\right)\right\}$.

For each $x=\left(x_{1}, x_{2}, \ldots\right) \in X$ and $n \geq 0$ define the row vector $s_{n}(x) \in \mathbb{N}^{d_{n}}$, called the suffix vector of order $n$ of $x$, by

$$
s_{n}(x, t)=\#\left\{e \in E_{n+1} ; x_{n+1} \preceq e, x_{n+1} \neq e, t \text { is the initial vertex of } e\right\}
$$

at each coordinate $t \in V_{n}$. A classical computation gives for all $n \geq 1$ (see for example [BDM05])

$$
r_{n}(x)=s_{0}(x)+\sum_{i=1}^{n-1}\left\langle s_{i}(x), h_{1} P_{i}\right\rangle=s_{0}(x)+\sum_{i=1}^{n-1}\left\langle s_{i}(x), h_{i}\right\rangle,
$$

where $\langle\cdot, \cdot\rangle$ is the euclidean inner product. Observe that under the hypothesis (H1), i.e., $h_{1}=(1, \ldots, 1)$, we have $s_{0}(x)=0$.

2.2.5. Invariant measures. Let $\mu$ be an invariant probability measure of the system $(X, T)$ associated to a properly ordered Bratteli diagram $B$, like in the previous subsection. It is determined by the values assigned to $B_{n}(t)$ for all $n \geq 0$ and $t \in V_{n}$. Define the column vector $\mu_{n}=\left(\mu_{n}(t) ; t \in V_{n}\right)$ with $\mu_{n}(t)=\mu\left(B_{n}(t)\right)$. A simple computation allows to prove the following useful relation:

$$
\mu_{m}=P_{m, n} \mu_{n}
$$

for integers $0 \leq m<n$. Also, $\mu\left(\tau_{n}=t\right)=h_{n}(t) \mu_{n}(t)$ for all $n \geq 1$ and $t \in V_{n}$.

2.2.6. Clean Bratteli-Vershik representations. Let $B$ be a proper ordered Bratteli diagram of finite rank $d$ and $(X, T)$ the corresponding minimal Cantor system. Recall that in this case we identify $V_{n}$ with $\{1, \ldots, d\}$ for all $n \geq 1$. Then, by Theorem 3.3 in [BKMS13], there exist a telescoping of the diagram (which keeps the diagram proper) and $\delta>0$ such that:

(1) For any ergodic measure $\mu$ there exists $I_{\mu} \subseteq\{1, \ldots, d\}$ verifying:

(a) $\mu\left(\tau_{n}=t\right) \geq \delta$ for every $t \in I_{\mu}$ and $n \geq 1$, and

(b) $\lim _{n \rightarrow \infty} \mu\left(\tau_{n}=t\right)=0$ for every $t \notin I_{\mu}$.

(2) If $\mu$ and $\nu$ are different ergodic measures then $I_{\mu} \cap I_{\nu}=\emptyset$.

When an ordered Bratteli diagram verifies the previous properties we say it is clean. We remark that this is a modified version of the notion of clean Bratteli diagram given in BDM10 that is inspired by the results of BKMS13. This property will be very relevant for formulating our main result. In BKMS13, systems such that $I_{\mu}=\{1, \ldots, d\}$ for some ergodic measure $\mu$ are called of exact finite rank. Those systems are uniquely ergodic.

Let $\lambda \in \mathbb{S}^{1}$ be an eigenvalue of the system $(X, T)$ associated to $B$ for an ergodic measure $\mu$. Let $f \in L^{2}(X, \mu)$ be an associated eigenfunction with $|f|=1$. For 
$n \geq 1$ define $c_{n}: V_{n} \rightarrow \mathbb{R}_{0}^{+}$and $\rho_{n}: V_{n} \rightarrow[0,1)$ by the relation

$$
\frac{1}{\mu_{n}(t)} \int_{B_{n}(t)} f d \mu=c_{n}(t) \lambda^{-\rho_{n}(t)}, \quad \text { for } \quad t \in V_{n}
$$

Notice that $0 \leq c_{n}(t) \leq 1$.

The sequence $\left(f_{n} ; n \geq 1\right)$ of conditional expectations of $f$ with respect to the sigma algebras $\left(\mathcal{T}_{n} ; n \geq 1\right)$ generated by the Kakutani-Rohlin partitions satisfies

$$
f_{n}(x)=\mathbb{E}\left(f \mid \mathcal{T}_{n}\right)(x)=c_{n}\left(\tau_{n}(x)\right) \lambda^{-r_{n}(x)-\rho_{n}\left(\tau_{n}(x)\right)} .
$$

It can be proved that $\lambda^{-\left(r_{n}+\rho_{n} \circ \tau_{n}\right)}$ converges $\mu$-a.e. (for a slightly deeper discussion we refer the reader to [BDM05]). Also, rephrasing a known result from BDM10] we have

Lemma 1. If $B$ is a clean Bratteli diagram and $\mu$ an ergodic measure for the associated minimal Cantor system, then

(1) for any $t \in\{1, \ldots, d\}, \lim _{n \rightarrow \infty} \mu\left(\tau_{n}=t\right)\left(c_{n}(t)-1\right) \rightarrow 0$,

(2) for $t \in I_{\mu}, \lim _{n \rightarrow \infty} c_{n}(t) \rightarrow 1$.

2.3. Bratteli-Vershik systems of Toeplitz type. A properly ordered Bratteli diagram $B=(V, E, \preceq)$ is of Toeplitz type if for all $n \geq 1$ the number of edges in $E_{n}$ finishing at a fixed vertex of $V_{n}$ is constant independently of the vertex. Denote this number by $q_{n}$ and set $p_{n}=q_{1} q_{2} \cdots q_{n}$. Observe that $p_{n}$ is the number of paths from $v_{0}$ to any vertex of $V_{n}$. Thus $h_{n}(t)=p_{n}$ for any $t \in V_{n}$. We say that $\left(q_{n} ; n \geq 1\right)$ is the characteristic sequence of the diagram. This class was obtained in GJ00 when characterizing Toeplitz subshifts.

The main object in this study are eigenvalues of minimal Cantor systems of finite rank $d$, having a proper Bratteli-Vershik representation of Toeplitz type. It is known that finite rank minimal Cantor systems are either odometers or subshifts DM08, so in our study we will be dealing only with Toeplitz subshifts or odometers.

To state our main results we will need some extra notations. Fix a minimal Cantor system $(X, T)$ with a Toeplitz type proper Bratteli-Vershik representation of rank $d$ and characteristic sequence $\left(q_{n} ; n \geq 1\right)$.

For $0 \leq m<n$ define $q_{m, n}=q_{m+1} \cdots q_{n}$, the number of paths in $E_{m, n}$ finishing in any fixed vertex $t \in V_{n}$. Clearly $q_{\ell, n}=q_{\ell, m} q_{m, n}$ if $0 \leq \ell<m<n$. Also, for $x=\left(x_{1}, x_{2}, \ldots\right) \in X$ define the integer $\bar{s}_{m, n}(x)$ as the number of paths in $E_{m, n}$ which end at $\tau_{n}(x)$ that are strictly bigger than $\left(x_{m+1}, \ldots, x_{n}\right)$ with respect to the induced partial order in $E_{m, n}$. Finally, define the set $\bar{S}_{m, n}\left(t_{1}, t_{2}\right)$ for $t_{1} \in V_{m}$ and $t_{2} \in V_{n}$ by

$$
\bar{S}_{m, n}\left(t_{1}, t_{2}\right)=\left\{\bar{s}_{m, n}(x) ; \tau_{m}(x)=t_{1} \text { and } \tau_{n}(x)=t_{2}\right\} .
$$

It is not difficult to prove that the cardinality of $\bar{S}_{m, n}\left(t_{1}, t_{2}\right)$ is equal to $P_{m, n}\left(t_{1}, t_{2}\right)$, that is, the number of paths from $t_{1} \in V_{m}$ to $t_{2} \in V_{n}$.

If necessary, to simplify notations we will denote $\bar{S}_{n, n+1}\left(t_{1}, t_{2}\right)$ by $\bar{S}_{n}\left(t_{1}, t_{2}\right)$ and $\bar{s}_{n, n+1}$ by $\bar{s}_{n}$. Notice that $\bar{s}_{n}(x)=\left\langle s_{n}(x),(1, \ldots, 1)\right\rangle=\sum_{t \in V_{n}} s_{n}(x, t)$ for any $x \in X$. 
We will need the following simple relations. For $0 \leq \ell<m<n, t_{1} \in V_{\ell}$ and $x \in X$ the following equalities hold:

$$
\begin{aligned}
r_{\ell}(x) & =\bar{s}_{0}(x)+\sum_{i=1}^{\ell-1} p_{i} \bar{s}_{i}(x), \\
\bar{s}_{\ell, m}(x) & =\bar{s}_{\ell}(x)+\sum_{i=\ell+1}^{m-1} q_{\ell+1} q_{\ell+2} \cdots q_{i} \bar{s}_{i}(x) \\
& =\frac{r_{m}(x)-r_{\ell}(x)}{p_{\ell}} \\
\bar{s}_{\ell, n}(x) & =\bar{s}_{\ell, m}(x)+q_{\ell, m} \bar{s}_{m, n}(x), \\
B_{\ell}\left(t_{1}\right) & =\bigcup_{t_{2} \in V_{m}} \bigcup_{s \in \bar{S}_{\ell, m}\left(t_{1}, t_{2}\right)} T^{-p_{\ell} s} B_{m}\left(t_{2}\right),
\end{aligned}
$$

where the union in the right hand side is disjoint.

\section{Eigenvalues of Toeplitz Systems of Finite Rank}

As was mentioned in the introduction, any countable subgroup of $\mathbb{S}^{1}=\{z \in$ $\mathbb{C} ;|z|=1\}$ containing infinitely many rationals can be the set of eigenvalues of a Toeplitz subshift for a given invariant measure Iwa96, DL96. Also, $\exp (2 i \pi \alpha) \in \mathbb{S}^{1}$ is a continuous eigenvalue of a minimal Cantor system with a Toeplitz type proper Bratteli-Vershik representation if and only if $\alpha=a / p_{m}$ for some $a \in \mathbb{Z}$ and $m \geq 1$ Wil84, JK69. A direct proof can be given using the particular combinatorial structure of the Brattelli-Vershik representation of a minimal Cantor system of Toeplitz type. We sketch it here. Using (2.4) and the fact that $p_{m}$ divides $p_{n}$ when $m \leq n$, one gets that $r_{n}(x) / p_{m}=\left(\bar{s}_{0}(x)+\sum_{i=1}^{m-1} p_{i} \bar{s}_{i}(x)\right) / p_{m} \bmod \mathbb{Z}$, for all $n \geq m$. Hence, $\exp \left(2 i \pi r_{n}(x) / p_{m}\right)$ converges uniformly when $n \rightarrow \infty$, which is a necessary and sufficient condition for $\exp \left(2 i \pi / p_{m}\right)$, and thus $\exp \left(2 i \pi a / p_{m}\right)$ for every $a \in \mathbb{Z}$, to be continuous eigenvalues in this context (see Proposition 12 in BDM05).

In the opposite direction, using the same criterion, if $\exp (2 i \pi / b)$ with $b \in \mathbb{Z}$ is a continuous eigenvalue, then $\left(r_{n+1}(x)-r_{n}(x)\right) / b=p_{n} \bar{s}_{n}(x) / b \bmod \mathbb{Z}$ is close to 0 for any large enough $n \geq 1$ and uniformly in $x$. Taking a point $x$ such that $\bar{s}_{n}(x)=1$ allows to conclude that $1 / b=a / p_{n}$ for some large $n \geq 1$ and $a \in \mathbb{Z}$. More details about continuous eigenvalues of Toeplitz type Bratteli-Vershik systems can be found in BDM10.

In the class of minimal Cantor systems with a Toeplitz type representation, the assumption of finite topological rank restricts the possibilities for non continuous eigenvalues. But, importantly, all are rational. In addition, if the characteristic sequence of a proper representation is bounded (or equivalently, a proper representation gives a linearly recurrent system), then all the eigenvalues are continuous. The following theorem gives a very restrictive condition verified by non continuous eigenvalues of Toeplitz systems in the finite rank case that are not linearly recurrent.

Theorem 2. BDM10 Let $(X, T)$ be a minimal Cantor system with a Toeplitz type proper Bratteli-Vershik representation of rank $d$ and characteristic sequence $\left(q_{n} ; n \geq 1\right)$. Let $\mu$ be an ergodic probability measure. If $\exp (2 i \pi a / b)$, with $(a, b)=1$, 
is a non continuous rational eigenvalue of $(X, T)$ for $\mu$, then $b /\left(b, p_{n}\right) \leq d$ for all $n$ large enough.

Let $\lambda=\exp (2 i \pi a / b)$, with $a, b$ integers such that $(a, b)=1$, be a non continuous rational eigenvalue as in the previous theorem. We notice that $b /\left(b, p_{n}\right)>1$ for all $n$ large enough. Indeed, if $b /\left(b, p_{n}\right)=1$ for some $n \geq 1$, then $1 / b=a^{\prime} / p_{n}$ for some $a^{\prime} \in \mathbb{Z}$, which by the discussion above implies that $\exp (2 i \pi a / b)$ is a continuous eigenvalue. Also, observe that $\left(b, p_{n}\right)$ is a non decreasing sequence of integers bounded by $b$, so $b /\left(b, p_{n}\right)$ is eventually constant, say equal to $\mathbf{b}$. Since we are considering proper representations, the fact that $\mathbf{b}>1$ implies that $\left(q_{n} ; n \geq 1\right)$ tends to infinity with $n$. Otherwise, the system will be linearly recurrent, and thus all eigenvalues will be continuous, which implies that $b /\left(b, p_{n}\right)=1$ for some $n>1$.

Now we state our main result,

Theorem 3. Let $(X, T)$ be a minimal Cantor system with a Toeplitz type proper and clean Bratteli-Vershik representation of rank $d$ and characteristic sequence $\left(q_{n} ; n \geq\right.$ $1)$. Let $\mu$ be an ergodic probability measure. Then, $\lambda=\exp (2 i \pi a / b)$, with $a, b$ integers such that $(a, b)=1$, is a non continuous eigenvalue of $(X, T)$ for $\mu$ if and only if

(1) $b /\left(b, p_{n}\right)=\mathbf{b}$ for all $n$ large enough and some $1<\mathbf{b} \leq d$, and

(2) for all $t_{2} \in I_{\mu}$

$$
\sum_{t_{1} \in V_{m}} \frac{\left|\sum_{s \in \bar{S}_{m, n}\left(t_{1}, t_{2}\right)} \lambda^{-p_{m} s}\right|}{q_{m, n}} \underset{m, n \rightarrow \infty}{\longrightarrow} 1,
$$

uniformly in $m, n \in \mathbb{N}$ with $m<n$.

As was mentioned in the introduction, even if this condition looks "heavy" to check, in fact it is easy to verify and construct examples fulfilling it. This will be illustrated in Section 6. The main tool is provided by the following corollary that follows from the construction in the proof of Theorem 3 .

Corollary 4. Let $(X, T)$ be a minimal Cantor system with a Toeplitz type proper and clean Bratteli-Vershik representation of rank $d$ and characteristic sequence $\left(q_{n} ; n \geq 1\right)$. Let $\mu$ be an ergodic probability measure. Let $\left(q_{n} ; n \geq 1\right)$ be its characteristic sequence. Then, $\lambda=\exp (2 i \pi a / b)$, with $a, b$ integers such that $(a, b)=1$, is a non continuous eigenvalue of $(X, T)$ for $\mu$ if and only if up to a telescoping of the diagram we have

(1) $p_{n}=p \bmod b$ for some $p \in\{0, \ldots, b-1\}$ and for all $n \geq 2$,

(2) $b /\left(b, p_{n}\right)=\mathbf{b}$ for all $n$ large enough and some $1<\mathbf{b} \leq d$,

(3) there exists a map $k(\cdot, \cdot):\{1, \ldots, d\} \times\{1, \ldots, d\} \rightarrow\{0, \ldots, \mathbf{b}-1\}$ such that

$$
\begin{aligned}
& p \cdot k\left(t_{1}, t_{3}\right)=p \cdot k\left(t_{1}, t_{2}\right)+p \cdot k\left(t_{2}, t_{3}\right) \bmod b, \\
& p \cdot k\left(t_{1}, t_{1}\right)=0 \quad \bmod b, \quad p \cdot k\left(t_{1}, t_{2}\right)=-p \cdot k\left(t_{2}, t_{1}\right) \bmod b,
\end{aligned}
$$

for all $t_{1}, t_{2}, t_{3} \in I_{\mu}$,

(4) for $\mu$-almost every point $x \in X$ the equality $\bar{s}_{n}(x)=k\left(\tau_{n}(x), \tau_{n+1}(x)\right)$ $\bmod \mathbf{b}$ holds for all large enough $n \in \mathbb{N}$. 
In what follows we provide a number of reformulations and corollaries of the main theorem. Some proofs are left to the reader since they can be easily deduced from a direct computation or Lemmas 12 and 13 provided below, others will be proved near the end of Section 5 after proving the main theorem.

We start by a natural reformulation of Theorem 3 . It says that we can replace $V_{m}$ by $I_{\mu}$ in the sum of statement (2) of the theorem. In other words, we only need to consider the vertices of the diagram determining the measure $\mu$. We will need the following observation: for $t_{1} \notin I_{\mu}$ and $t_{2} \in I_{\mu}$ one has

$$
\frac{P_{m, n}\left(t_{1}, t_{2}\right)}{q_{m, n}} \underset{m, n \rightarrow \infty}{\longrightarrow} 0
$$

uniformly in $m, n \in \mathbb{N}$ with $m<n$. Indeed, since the diagram is clean, $\mu\left(\tau_{n}=\right.$ $\left.t_{2}\right) \geq \delta>0$ and $\lim _{m \rightarrow \infty} \mu\left(\tau_{m}=t_{1}\right)=0$. These facts, together with the following inequalities

$$
\frac{P_{m, n}\left(t_{1}, t_{2}\right)}{q_{m, n}} \cdot \delta \leq \frac{P_{m, n}\left(t_{1}, t_{2}\right)}{q_{m, n}} \mu\left(\tau_{n}=t_{2}\right)=\mu\left(\tau_{m}=t_{1}, \tau_{n}=t_{2}\right) \leq \mu\left(\tau_{m}=t_{1}\right),
$$

allow to deduce (3.1). Since the cardinality of $\bar{S}_{m, n}\left(t_{1}, t_{2}\right)$ is equal to $P_{m, n}\left(t_{1}, t_{2}\right)$, we also deduce that

$$
\sum_{t_{1} \in V_{m} \backslash I_{\mu}} \frac{\left|\sum_{s \in \bar{S}_{m, n}\left(t_{1}, t_{2}\right)} \lambda^{-p_{m} s}\right|}{q_{m, n}} \leq \sum_{t_{1} \in V_{m} \backslash I_{\mu}} \frac{P_{m, n}\left(t_{1}, t_{2}\right)}{q_{m, n}} .
$$

Therefore, a direct application of (3.1) in the last inequality allows to reformulate Theorem 3 as follows.

Corollary 5 (Variation on Theorem 3). The complex number $\lambda=\exp (2 i \pi a / b)$, with $a, b$ integers such that $(a, b)=1$, is a non continuous eigenvalue of $(X, T)$ for $\mu$ if and only if

(1) $b /\left(b, p_{n}\right)=\mathbf{b}$ for all $n$ large enough and some $1<\mathbf{b} \leq d$, and

(2) for all $t_{2} \in I_{\mu}$

$$
\sum_{t_{1} \in I_{\mu}} \frac{\left|\sum_{s \in \bar{S}_{m, n}\left(t_{1}, t_{2}\right)} \lambda^{-p_{m} s}\right|}{q_{m, n}} \underset{m, n \rightarrow \infty}{\longrightarrow} 1,
$$

uniformly in $m, n \in \mathbb{N}$ with $m<n$.

The following corollary is a reformulation of the main condition of Theorem 3 and the corresponding one in Corollary 5 . It follows almost directly by combining Lemmas 12 and 13 in the next section, so its proof is left to the reader.

Corollary 6. The main condition in Theorem 3 (resp. Corollary 5) is equivalent to: for all $t_{2} \in I_{\mu}$ and $m \geq 1$ there exists a sequence of partitions $\left(\mathcal{H}_{m, n, t_{2}} ; m<n\right)$ of $V_{m}$ (resp. of $I_{\mu}$ ) with $\# \mathcal{H}_{m, n, t_{2}}=\mathbf{b}$ such that

$$
\sum_{t_{1} \in A} \frac{\left|\sum_{s \in \bar{S}_{m, n}\left(t_{1}, t_{2}\right)} \lambda^{-p_{m} s}\right|}{q_{m, n}} \underset{m, n \rightarrow \infty}{\longrightarrow} \frac{1}{\mathbf{b}},
$$

uniformly in $m, n \in \mathbb{N}$ with $m<n$ for any $A \in \mathcal{H}_{m, n, t_{2}}$. 
This formulation pinpoints to the possible local orders that accept a BratteliVershik representation to have non continuous eigenvalues. Part (3) of Lemma 12 states that the main condition of Theorem 3 (or its equivalent formulations) implies that the local order of most of the arrows from a vertex in an atom $A \in \mathcal{H}_{m, n, t_{2}}$ to $t_{2} \in I_{\mu}$ at level $n$ must be congruent modulo $\mathbf{b}$. This condition is one of the main tools to explore non continuous rational eigenvalues of Toeplitz systems.

Another interesting fact is that we can relate non continuous eigenvalues with the number of ergodic invariant measures of a Toeplitz system. Let $(X, T)$ be a minimal Cantor system and $\mu$ an ergodic measure as in Theorem 3. Define,

$$
\mathbf{B}_{\mu}=\left\{\lim _{m \rightarrow \infty} b /\left(b, p_{m}\right) ; b \in \mathbb{N}, \exp (2 i \pi / b) \text { is a non continuous eigenvalue for } \mu\right\}
$$

and endow it with the divisibility (partial) order. Recall that $\lim _{m \rightarrow \infty} b /\left(b, p_{m}\right)$ is equal to $\mathbf{b}=b /\left(b, p_{n}\right)$ for a large $n \in \mathbb{N}$. Denote by $\mathcal{M}_{\text {erg }}(X, T)$ the set of ergodic measures of $(X, T)$ and consider the set $\mathcal{M}$ defined by:

$$
\mathcal{M}=\left\{\mu \in \mathcal{M}_{\text {erg }}(X, T) ; \mathbf{B}_{\mu} \neq \emptyset\right\} .
$$

Corollary 7. The following properties hold:

(1) For any $\mu \in \mathcal{M}$ and $\mathbf{b} \in \mathbf{B}_{\mu}, \mathbf{b} \leq \# I_{\mu}$.

(2) For any $\mu \in \mathcal{M}, \mathbf{B}_{\mu}$ has a unique divisibility-maximal element $\mathbf{b}_{\mu}$.

(3) $\sum_{\mu \in \mathcal{M}} \mathbf{b}_{\mu} \leq d$.

(4) $\# \mathcal{M} \leq \# \mathcal{M}_{\text {erg }}(X, T) \leq d-\sum_{\mu \in \mathcal{M}}\left(\mathbf{b}_{\mu}-1\right)$.

The proof of this corollary will be given at the end of Section 5 ,

Fix an ergodic measure $\mu$. To understand better the last corollary let us suppose the $p_{n}$ 's are powers of the same prime number. In this case, for all integers $b$ such that $\lambda=\exp (2 i \pi / b)$ is a non continuous eigenvalue for $\mu$ one has $\left(b, p_{n}\right)=1$ and parts (1) and (2) of last corollary tell us that there is a unique $b=\mathbf{b}_{\mu} \leq \# I_{\mu} \leq d$ which is maximal in $\mathbf{B}_{\mu}$. All other non continuous eigenvalues for $\mu$ are powers of $\lambda$. If $\mathbf{B}_{\mu}$ is empty, no non continuous eigenvalues exist for $\mu$. Notice that property (1) implies that we need at least $\mathbf{b}_{\mu}$ vertices to have the non continuous eigenvalue $\lambda$. Since $I_{\mu} \cap I_{\nu}=\emptyset$ for different ergodic measures $\mathbf{b}_{\nu} \leq d-\# I_{\mu} \leq d-\mathbf{b}_{\mu}$. We will see in some examples of Section 6 that these inequalities can be strict.

In the particular case when $\mathbf{b}_{\mu}=d$ for some ergodic measure $\mu$ we get the following corollary.

Corollary 8. Consider $\lambda=\exp (2 i \pi a / b)$, with $a, b$ integers such that $(a, b)=1$ and $b /\left(b, p_{n}\right)=d$ for all $n$ large enough. Then $\lambda$ is a non continuous eigenvalue of $(X, T)$ for the invariant measure $\mu$ if and only if for all $t_{1}, t_{2} \in\{1, \ldots, d\}$

$$
\frac{\left|\sum_{s \in \bar{S}_{m, n}\left(t_{1}, t_{2}\right)} \lambda^{-p_{m} s}\right|}{q_{m, n}} \underset{m, n \rightarrow \infty}{\longrightarrow} \frac{1}{d}
$$

uniformly in $m, n \in \mathbb{N}$ with $m<n$. If $\lambda$ is an eigenvalue, then:

(1) the system $(X, T)$ is uniquely ergodic and $\mu$ is the unique invariant measure,

(2) for all $t \in\{1, \ldots, d\}, \lim _{n \rightarrow \infty} \mu\left(\tau_{n}=t\right)=1 / d$. 
Condition (3.2) and statement (11) follow almost directly from Corollaries 6 and 7 Nevertheless, we provide a complete proof of the corollary at the end of Section 5 .

An analogous result to Corollary 8 can be obtained when the system is uniquely ergodic and $b /\left(b, p_{n}\right)=\# I_{\mu}$ for all $n$ large enough. The statement is obtained by replacing $d$ by $\# I_{\mu}$ and the set $\{1, \ldots, d\}$ by $I_{\mu}$ in the last corollary.

\section{MAin TeChNiCAL LEMMAS}

In this section we will provide the main ingredients we need to prove Theorem 3 and its corollaries.

4.1. A geometric lemma. The next lemma can be stated in a much more general situation and its proof follows from general facts of convex analysis. Nevertheless, since we consider a particular case, we provide a simple self-contained proof.

Lemma 9. Let $N$ be a positive integer. Then there exists a constant $C$ such that for any convex combination $w=\sum_{j=0}^{N-1} \alpha_{j} \xi^{j}$ of the $N$-th roots of unity $1, \xi, \ldots, \xi^{N-1}$ verifying $1-\varepsilon<|w| \leq 1$ for some $\varepsilon>0$ one has

$$
1-C \varepsilon<\alpha_{i} \leq 1
$$

for some $0 \leq i \leq N-1$.

Proof. A proof is given only in the case when $|w| \neq 1$. Write $w$ in the following way

$$
w=\alpha_{i} \xi^{i}+\beta \zeta,
$$

where $\alpha_{i} \geq 1 / N$ and $\alpha_{i}+\beta=1$ (note that $\zeta$ belongs to the convex hull of the $N$-th roots of unity different from $\xi^{i}$ ). The function $F(z)=\alpha_{i} \xi^{i}+\beta z$ has maximal absolute value at $z \in\left\{\xi^{i-1}, \xi^{i+1}\right\}$ when restricted to the convex hull of the $N$-th roots of unity different from $\xi^{i}$. Hence

$$
\begin{aligned}
1-\varepsilon & <|w| \quad(=|F(\zeta)|) \\
& \leq\left|F\left(\xi^{i+1}\right)\right| \\
& =|1+\beta(\xi-1)| \\
& =\sqrt{1-2 \beta(1-\beta)(1-\cos 2 \pi / N)} \\
& \leq 1-\beta(1-\beta)(1-\cos 2 \pi / N) \\
& \leq 1-\beta\left(\frac{1-\cos (2 \pi / N)}{N}\right)
\end{aligned}
$$

and

$$
\alpha_{\ell}>1-\left(\frac{N}{1-\cos (2 \pi / N)}\right) \varepsilon
$$

4.2. Special telescoping of a Bratteli-Vershik system. At some point of the proof of Theorem 3 we will need to telescope an ordered Bratteli diagram in the following particular way. 
Lemma 10. Let $B=(V, E, \preceq)$ be an ordered Bratteli diagram such that $\# V_{n}=d$ for all $n \geq 1$ and identify $V_{n}$ with $\{1, \ldots, d\}$. For all $1 \leq m<n$ and $t \in\{1, \ldots, d\}$ consider $\left(\mathcal{G}_{m, n, t}, \leq_{m, n, t}\right)$, where $\mathcal{G}_{m, n, t}$ is a partition of $V_{m}$ and $\leq_{m, n, t}$ is a total ordering on the atoms of $\mathcal{G}_{m, n, t}$. Then, there exists a strictly increasing sequence $\left(n_{k}\right)_{k \geq 0}$ in $\mathbb{N}$ such that for all $k_{0} \geq 0, k>k_{0}$ and $t \in\{1, \ldots, d\}$ we have

$$
\left(\mathcal{G}_{n_{k_{0}}, n_{k}, t}, \leq_{n_{k_{0}}, n_{k}, t}\right)=\left(\mathcal{G}_{n_{k_{0}}, n_{k+1}, t}, \leq_{n_{k_{0}}, n_{k+1}, t}\right) .
$$

Proof. It suffices to remark that there are finitely many such structures on $\{1, \ldots, d\}$ (partitions endowed with total orderings). Then, one proceeds by induction using the pigeon hole principle.

Let us give some details. Take $n_{0}=1$. By the pigeon hole principle, there exists a strictly increasing sequence $\left(n_{k}^{(0)}\right)_{k \geq 0}$, with $n_{0}^{(0)}>n_{0}$, such that for all $k \geq 0$ and $t \in\{1, \ldots, d\}$ we have

$$
\left(\mathcal{G}_{n_{0}, n_{k}^{(0)}, t}, \leq_{n_{0}, n_{k}^{(0)}, t}\right)=\left(\mathcal{G}_{n_{0}, n_{k+1}^{(0)}, t}, \leq_{n_{0}, n_{k+1}^{(0)}, t}\right) .
$$

Now, let $n_{1}=n_{0}^{(0)}$. Using the same argument, there exists a strictly increasing subsequence $\left(n_{k}^{(1)}\right)_{k \geq 0}$ of $\left(n_{k}^{(0)}\right)_{k \geq 0}$, with $n_{0}^{(1)}>n_{0}^{(0)}$, such that for all $k \geq 0$ and $t \in\{1, \ldots, d\}$ we have $\left(\mathcal{G}_{n_{1}, n_{k}^{(1)}, t}, \leq_{n_{1}, n_{k}^{(1)}, t}\right)=\left(\mathcal{G}_{n_{1}, n_{k+1}^{(1)}, t}, \leq_{n_{1}, n_{k+1}^{(1)}, t}\right)$. Observe that we also have $\left(\mathcal{G}_{n_{0}, n_{k}^{(1)}, t}, \leq_{n_{0}, n_{k}^{(1)}, t}\right)=\left(\mathcal{G}_{n_{0}, n_{k+1}^{(1)}, t}, \leq_{n_{0}, n_{k+1}^{(1)}, t}\right)$ for all $k \geq 0$ and $t \in$ $\{1, \ldots, d\}$ by construction. Proceeding in this way we obtain the desired sequence $\left(n_{k}\right)_{k \geq 0}$.

\subsection{Uniform lower bound for consecutive towers in $I_{\mu}$.}

Lemma 11. Let $(X, T)$ be a minimal Cantor system with a Toeplitz type proper and clean Bratteli-Vershik representation of rank $d$ and $\mu$ be an ergodic probability measure. Let $\left(q_{n} ; n \geq 1\right)$ be its characteristic sequence. For all $m \geq 1$, there exists $n_{0}>m$ such that for all $n \geq n_{0}$ and $t_{1}, t_{2} \in I_{\mu}$

$$
\frac{P_{m, n}\left(t_{1}, t_{2}\right)}{q_{m, n}} \geq \frac{\delta}{3},
$$

where $\delta>0$ is such that $\mu\left(\tau_{n}=t\right) \geq \delta$ for any $t \in I_{\mu}$ and $n \in \mathbb{N}$ (coming from the cleanliness property of the diagram).

Proof. Fix $m \geq 1$ and $0<\epsilon<\delta^{2} / 3$. From Egorov's theorem and the ergodic theorem, there exists a measurable subset $A_{\epsilon}$ with $\mu\left(A_{\epsilon}\right) \geq 1-\epsilon$ and a positive integer $M_{0}$ such that for all $x \in A_{\epsilon}$ and $M \geq M_{0}$ we have

$$
\left|\frac{1}{M} \sum_{k=0}^{M-1} 1_{\left\{\tau_{m}=t_{1}\right\}}\left(T^{k} x\right)-\mu\left(\tau_{m}=t_{1}\right)\right|<\epsilon .
$$

Let $n>m$ be such that $p_{n} \geq M_{0}$ (recall that $p_{n}$ is the number of paths from $v_{0}$ to any vertex of $\left.V_{n}\right)$. There exists $x \in A_{\epsilon} \cap T^{-p_{n}-j+1} B_{n}\left(t_{2}\right)$ for some $0 \leq j \leq\left\lfloor\frac{\epsilon p_{n}}{\delta}\right\rfloor<$ $p_{n}$. Indeed,

$$
\mu\left(\bigcup_{j=0}^{\left\lfloor\frac{\epsilon p_{n}}{\delta}\right\rfloor} T^{-\left(p_{n}+j-1\right)} B_{n}\left(t_{2}\right)\right)=\left(\left\lfloor\frac{\epsilon p_{n}}{\delta}\right\rfloor+1\right) \mu\left(B_{n}\left(t_{2}\right)\right)>\frac{\epsilon}{\delta} \mu\left(\tau_{n}=t_{2}\right) \geq \epsilon,
$$

since $\mu\left(\tau_{n}=t_{2}\right)=p_{n} \mu\left(B_{n}\left(t_{2}\right)\right)$ and $t_{2} \in I_{\mu}$. Hence, $\bigcup_{j=0}^{\left\lfloor\frac{\epsilon p_{n}}{\delta}\right\rfloor} T^{-\left(p_{n}+j-1\right)} B_{n}\left(t_{2}\right)$ must intersect $A_{\epsilon}$. Notice that the iterates $T^{j} x, \ldots, T^{j+p_{n}-1} x$ cross completely 
tower $t_{2} \in V_{n}$, from the lowest to the highest level. So those iterates enter to tower $t_{1} \in V_{m}$ exactly $P_{m, n}\left(t_{1}, t_{2}\right) p_{m}$ times.

Then, since $t_{1} \in I_{\mu}, p_{n}+j \geq M_{0}$ and $x \in A_{\epsilon}$, we can use (4.1) to get

$$
\begin{aligned}
\delta-\epsilon & \leq \mu\left(\tau_{m}=t_{1}\right)-\epsilon \leq \frac{1}{p_{n}+j} \sum_{k=0}^{p_{n}+j-1} 1_{\left\{\tau_{m}=t_{1}\right\}}\left(T^{k} x\right) \\
& \leq \frac{j}{p_{n}+j}+\frac{1}{p_{n}+j} \sum_{k=0}^{p_{n}-1} 1_{\left\{\tau_{m}=t_{1}\right\}}\left(T^{k}\left(T^{j} x\right)\right) \\
& \leq \frac{\epsilon}{\delta}+\frac{P_{m, n}\left(t_{1}, t_{2}\right) p_{m}}{p_{n}+j} \leq \frac{\epsilon}{\delta}+\frac{P_{m, n}\left(t_{1}, t_{2}\right)}{q_{m, n}} \leq \frac{\delta}{3}+\frac{P_{m, n}\left(t_{1}, t_{2}\right)}{q_{m, n}}
\end{aligned}
$$

which ends the proof.

4.4. Equivalent conditions for Theorem 3. We follow the same notations as in Theorem 3, $\lambda=\exp (2 i \pi a / b)$, with $(a, b)=1$, and $\mathbf{b}$ is the limit in $n$ of $b /\left(b, p_{n}\right)$, which is attained from some large $n \in \mathbb{N}$. In the sequel, equality modulo $\mathbf{b}$ and $b$ will be written $\equiv_{\mathbf{b}}$ and $\equiv_{b}$ respectively.

To make the text lighter, we need to introduce some extra notations. For $t_{1}, t_{2} \in$ $\{1, \ldots, d\}, k \in\{0, \ldots, \mathbf{b}-1\}$ and integers $1 \leq m<n$, set

$$
\begin{aligned}
\sigma_{m, n}\left(t_{1}, t_{2}\right) & =\sum_{s \in \bar{S}_{m, n}\left(t_{1}, t_{2}\right)} \lambda^{-p_{m} s} \\
\boldsymbol{\sigma}_{m, n}^{(k)}\left(t_{1}, t_{2}\right) & =\#\left\{s \in \bar{S}_{m, n}\left(t_{1}, t_{2}\right) ; s \equiv_{\mathbf{b}} k\right\}
\end{aligned}
$$

Notice that for $s, s^{\prime} \in \bar{S}_{m, n}\left(t_{1}, t_{2}\right), \lambda^{-p_{m} s}=\lambda^{-p_{m} s^{\prime}}$ if and only if $s \equiv_{\mathbf{b}} s^{\prime}$. Then,

$$
\begin{aligned}
\sigma_{m, n}\left(t_{1}, t_{2}\right) & =\sum_{k=0}^{\mathbf{b}-1} \lambda^{-p_{m} k} \boldsymbol{\sigma}_{m, n}^{(k)}\left(t_{1}, t_{2}\right), \\
P_{m, n}\left(t_{1}, t_{2}\right) & =\sum_{k=0}^{\mathbf{b}-1} \boldsymbol{\sigma}_{m, n}^{(k)}\left(t_{1}, t_{2}\right), \\
\left|\sigma_{m, n}\left(t_{1}, t_{2}\right)\right| & \leq P_{m, n}\left(t_{1}, t_{2}\right), \\
q_{m, n} & =\sum_{k=0}^{\mathbf{b}-1} \sum_{t_{1} \in V_{m}} \boldsymbol{\sigma}_{m, n}^{(k)}\left(t_{1}, t_{2}\right), \\
\sum_{t_{1} \in V_{m}} \boldsymbol{\sigma}_{m, n}^{(k)}\left(t_{1}, t_{2}\right) & =\left\lfloor\frac{q_{m, n}}{\mathbf{b}}\right\rfloor \text { or }\left\lfloor\frac{q_{m, n}}{\mathbf{b}}\right\rfloor+1 .
\end{aligned}
$$

Lemma 12. For any $t_{2} \in\{1, \ldots, d\}$ the following conditions are equivalent:

(1) $\sum_{t_{1} \in V_{m}} \frac{\left|\sigma_{m, n}\left(t_{1}, t_{2}\right)\right|}{q_{m, n}} \underset{m, n \rightarrow \infty}{\longrightarrow} 1 \quad$ uniformly in $m, n \in \mathbb{N}$ with $m<n$ (this is condition (2) of Theorem 3 stated for any $t_{2}$ ).

(2) For all $t_{1} \in\{1, \ldots, d\}$,

$$
\frac{\left|\sigma_{m, n}\left(t_{1}, t_{2}\right)\right|}{q_{m, n}}-\frac{P_{m, n}\left(t_{1}, t_{2}\right)}{q_{m, n}} \underset{m, n \rightarrow \infty}{\longrightarrow} 0
$$


uniformly in $m, n \in \mathbb{N}$ with $m<n$.

(3) For all integers $1 \leq m<n$ and $t_{1} \in\{1, \ldots, d\}$, there exists $k_{m, n}\left(t_{1}, t_{2}\right)$ in $\{0, \ldots, \mathbf{b}-1\}$ such that

$$
\frac{\boldsymbol{\sigma}_{m, n}^{\left(k_{m, n}\left(t_{1}, t_{2}\right)\right)}\left(t_{1}, t_{2}\right)}{q_{m, n}}-\frac{P_{m, n}\left(t_{1}, t_{2}\right)}{q_{m, n}} \underset{m, n \rightarrow \infty}{\longrightarrow} 0
$$

uniformly in $m, n \in \mathbb{N}$ with $m<n$.

Proof. (1) $\Longrightarrow(2)$. We proceed by contradiction. Suppose there exists $\overline{t_{1}} \in\{1, \ldots, d\}$ such that for infinitely many positive integers $m, n$ with $m<n$

$$
\frac{P_{m, n}\left(\overline{t_{1}}, t_{2}\right)}{q_{m, n}}-\frac{\left|\sigma_{m, n}\left(\bar{t}_{1}, t_{2}\right)\right|}{q_{m, n}} \geq 2 \varepsilon>0,
$$

where $\varepsilon$ is a positive real.

From (1) we have that for any large enough positive integers $m, n$ with $m<n$

$$
1-\varepsilon<\sum_{t_{1} \in V_{m}} \frac{\left|\sigma_{m, n}\left(t_{1}, t_{2}\right)\right|}{q_{m, n}}<1+\varepsilon
$$

Consider a pair of large integers $m, n$ with $m<n$ verifying (4.9). Then, from (4.6), (4.9) and (4.10) we get

$$
1=\sum_{t_{1} \in V_{m}} \frac{P_{m, n}\left(t_{1}, t_{2}\right)}{q_{m, n}} \geq 2 \varepsilon+\sum_{t_{1} \in V_{m}} \frac{\left|\sigma_{m, n}\left(t_{1}, t_{2}\right)\right|}{q_{m, n}} \geq 1+\varepsilon,
$$

which is impossible. Condition (2) follows.

$(2) \Longrightarrow(3)$. Take $\varepsilon>0$. By hypothesis and (4.6), there exists a positive integer $N$ such that for all $n>m>N$ and $t_{1} \in\{1, \ldots, d\}$

$$
0 \leq \frac{P_{m, n}\left(t_{1}, t_{2}\right)}{q_{m, n}}-\frac{\left|\sigma_{m, n}\left(t_{1}, t_{2}\right)\right|}{q_{m, n}}<\varepsilon .
$$

Alternatively, the last inequality can be written as

$$
1-\frac{\varepsilon q_{m, n}}{P_{m, n}\left(t_{1}, t_{2}\right)}<\left|\sum_{k=0}^{\mathbf{b}-1} \frac{\boldsymbol{\sigma}_{m, n}^{(k)}\left(t_{1}, t_{2}\right)}{P_{m, n}\left(t_{1}, t_{2}\right)} \lambda^{-k p_{m}}\right| \leq 1 .
$$

Notice that $\left\{1, \lambda^{-p_{m}}, \ldots, \lambda^{-(\mathbf{b}-1) p_{m}}\right\}$ is the complete set of $\mathbf{b}$-th roots of unity if $m$ is large enough, and we have a convex combination of them. Applying Lemma 9 we deduce that there exists $k_{m, n}\left(t_{1}, t_{2}\right) \in\{0, \ldots, \mathbf{b}-1\}$ such that

$$
1-\frac{C \varepsilon q_{m, n}}{P_{m, n}\left(t_{1}, t_{2}\right)}<\frac{\boldsymbol{\sigma}_{m, n}^{\left(k_{m, n}\left(t_{1}, t_{2}\right)\right)}\left(t_{1}, t_{2}\right)}{P_{m, n}\left(t_{1}, t_{2}\right)} \leq 1,
$$

or equivalently,

$$
0 \leq \frac{P_{m, n}\left(t_{1}, t_{2}\right)}{q_{m, n}}-\frac{\boldsymbol{\sigma}_{m, n}^{\left(k_{m, n}\left(t_{1}, t_{2}\right)\right)}\left(t_{1}, t_{2}\right)}{q_{m, n}}<C \varepsilon .
$$

The constructed sequence depends on $\varepsilon$. Taking a sequence $\left(\varepsilon_{\ell} ; \ell \in \mathbb{N}\right)$ tending to zero and using a diagonal process one obtains the desired sequence

$$
\left(k_{m, n}\left(t_{1}, t_{2}\right) ; m, n \in \mathbb{N}, m<n\right) .
$$


$(3) \Longrightarrow(1)$. Fix $\varepsilon>0$. There exists a positive integer $N$ large enough such that for any $n>m>N$ and $t_{1} \in\{1, \ldots, d\}$,

$$
0 \leq \frac{P_{m, n}\left(t_{1}, t_{2}\right)}{q_{m, n}}-\frac{\boldsymbol{\sigma}_{m, n}^{\left(k_{m, n}\left(t_{1}, t_{2}\right)\right)}\left(t_{1}, t_{2}\right)}{q_{m, n}}=\sum_{\substack{k=0 \\ k \neq k_{m, n}\left(t_{1}, t_{2}\right)}}^{\mathbf{b}-1} \frac{\boldsymbol{\sigma}_{m, n}^{(k)}\left(t_{1}, t_{2}\right)}{q_{m, n}}<\varepsilon .
$$

So, using relations (4.4) and (4.11) we deduce that

$$
\begin{aligned}
\left|\sigma_{m, n}\left(t_{1}, t_{2}\right)\right| & =\left|\boldsymbol{\sigma}_{m, n}^{\left(k_{m, n}\left(t_{1}, t_{2}\right)\right)}\left(t_{1}, t_{2}\right) \lambda^{-p_{m} k_{m, n}\left(t_{1}, t_{2}\right)}+\sum_{\substack{k=0 \\
k \neq k_{m, n}\left(t_{1}, t_{2}\right)}}^{\mathbf{b}-1} \boldsymbol{\sigma}_{m, n}^{(k)}\left(t_{1}, t_{2}\right) \lambda^{-p_{m} k}\right| \\
& \geq \boldsymbol{\sigma}_{m, n}^{\left(k_{m, n}\left(t_{1}, t_{2}\right)\right)}\left(t_{1}, t_{2}\right)-\sum_{\substack{k=0 \\
k \neq k_{m, n}\left(t_{1}, t_{2}\right)}}^{\mathbf{b}-1} \boldsymbol{\sigma}_{m, n}^{(k)}\left(t_{1}, t_{2}\right) \\
& \geq \boldsymbol{\sigma}_{m, n}^{\left(k_{m, n}\left(t_{1}, t_{2}\right)\right)}\left(t_{1}, t_{2}\right)-\epsilon q_{m, n} .
\end{aligned}
$$

From this inequality, (4.6) and (4.11) we get

$$
\frac{\boldsymbol{\sigma}_{m, n}^{\left(k_{m, n}\left(t_{1}, t_{2}\right)\right)}\left(t_{1}, t_{2}\right)}{q_{m, n}}-\varepsilon \leq \frac{\left|\sigma_{m, n}\left(t_{1}, t_{2}\right)\right|}{q_{m, n}} \leq \frac{\boldsymbol{\sigma}_{m, n}^{\left(k_{m, n}\left(t_{1}, t_{2}\right)\right)}\left(t_{1}, t_{2}\right)}{q_{m, n}}+\varepsilon .
$$

Finally, from (4.5) and (4.11) applied to these last inequalities we deduce that

$$
\frac{P_{m, n}\left(t_{1}, t_{2}\right)}{q_{m, n}}-2 \varepsilon \leq \frac{\left|\sigma_{m, n}\left(t_{1}, t_{2}\right)\right|}{q_{m, n}} \leq \frac{P_{m, n}\left(t_{1}, t_{2}\right)}{q_{m, n}}+\varepsilon .
$$

Adding over $t_{1} \in V_{m}$ we get

$$
\left|\sum_{t_{1} \in V_{m}} \frac{\left|\sigma_{m, n}\left(t_{1}, t_{2}\right)\right|}{q_{m, n}}-1\right| \leq 2 d \varepsilon
$$

Property (1) follows since this inequality is valid for any $n>m>N$ given $\varepsilon>$ 0.

Notice that the sequence $\left(k_{m, n}\left(t_{1}, t_{2}\right) ; m, n \in \mathbb{N}, m<n\right)$ in statement (3) of Lemma 12 is not necessarily uniquely defined.

4.5. Constructing a partition from Theorem 3. The next lemma allows to construct several partitions of the vertices in a level of the Bratteli diagram such that the local order of most of the arrows starting in a vertex of an atom of such partition ending in the same vertex of a further level must be congruent modulo $\mathbf{b}$. This is crucial to get Corollary 6 .

Lemma 13. For $t_{2} \in\{1, \ldots, d\}$ asssume that any of the equivalent conditions in Lemma 12 holds. For each $t_{1} \in\{1, \ldots, d\}$ fix a sequence $\left(k_{m, n}\left(t_{1}, t_{2}\right) ; m, n \in\right.$ $\mathbb{N}, m<n)$ as in statement (3) of Lemma 12. Consider the map

$$
\begin{aligned}
\Psi_{m, n, t_{2}}:\{1, \ldots, d\} & \rightarrow\{0, \ldots, \mathbf{b}-1\} \\
t_{1} & \mapsto k_{m, n}\left(t_{1}, t_{2}\right)
\end{aligned}
$$

Then,

(1) for any large enough $m, n \in \mathbb{N}$ with $m<n, \Psi_{m, n, t_{2}}$ is onto, 
(2) for any $k \in\{0, \ldots, \mathbf{b}-1\}$,

$$
\sum_{t_{1} \in \Psi_{m, n, t_{2}}^{-1}(k)} \frac{\boldsymbol{\sigma}_{m, n}^{(k)}\left(t_{1}, t_{2}\right)}{q_{m, n}} \underset{m, n \rightarrow \infty}{\longrightarrow} \frac{1}{\mathbf{b}}
$$

uniformly in $m, n \in \mathbb{N}$ with $m<n$.

Proof. (1) Fix $0<\varepsilon<1 /(d+1)^{2}$. For any $t_{1} \in\{1, \ldots, d\}$ we have

$$
\frac{P_{m, n}\left(t_{1}, t_{2}\right)}{q_{m, n}}-\frac{\boldsymbol{\sigma}_{m, n}^{\left(k_{m, n}\left(t_{1}, t_{2}\right)\right)}\left(t_{1}, t_{2}\right)}{q_{m, n}}=\sum_{\substack{k=1 \\ k \neq k_{m, n}\left(t_{1}, t_{2}\right)}}^{\mathbf{b}-1} \frac{\boldsymbol{\sigma}_{m, n}^{(k)}\left(t_{1}, t_{2}\right)}{q_{m, n}} .
$$

Then, since by hypothesis $t_{2}$ verifies condition (3) of Lemma 12 for any $m, n \in \mathbb{N}$ with $m<n$ large enough $\frac{\boldsymbol{\sigma}_{m, n}^{(k)}\left(t_{1}, t_{2}\right)}{q_{m, n}}<\varepsilon$ for all $t_{1} \in\{1, \ldots, d\}$ and $k \neq k_{m, n}\left(t_{1}, t_{2}\right)$. Since $q_{n}$ goes to infinity with $n$, then considering larger values of $m, n$ we can also assume that $1 / q_{m, n}<\varepsilon$.

If assertion (1) of the lemma is not true, then for some large $m, n$ with $m<n$, there is $k \in\{0, \ldots, \mathbf{b}-1\} \backslash \operatorname{Im} \Psi_{m, n, t_{2}}$. Hence, by the previous considerations and equality (4.8)

$$
\frac{1}{\mathbf{b}}-\varepsilon<\sum_{t_{1} \in V_{m}} \frac{\boldsymbol{\sigma}_{m, n}^{(k)}\left(t_{1}, t_{2}\right)}{q_{m, n}}<d \varepsilon,
$$

which, by the choice of $\varepsilon$, contradicts the fact that $\mathbf{b} \leq d$.

(2) Fix $\varepsilon>0$. By part (1), there exists $N \in \mathbb{N}$ such that for all $n>m>N, \Psi_{m, n, t_{2}}$ is surjective. Taking a larger $N$ if necessary we can also assume that $1 / q_{m, n}$ and $\frac{\boldsymbol{\sigma}_{m, n}^{(k)}\left(t_{1}, t_{2}\right)}{q_{m, n}}$ are less than $\varepsilon$ for all $t_{1} \in\{1, \ldots, d\}$ and $k \neq k_{m, n}\left(t_{1}, t_{2}\right)$.

Let $k$ be an element in $\{0, \ldots, \mathbf{b}-1\}$. By (4.8) the following inequalities hold for all $n>m>N$ :

$$
\begin{aligned}
& \frac{1}{\mathbf{b}}-\varepsilon<\sum_{t_{1} \in V_{m}} \frac{\boldsymbol{\sigma}_{m, n}^{(k)}\left(t_{1}, t_{2}\right)}{q_{m, n}} \leq \frac{1}{\mathbf{b}}+\varepsilon \\
& \frac{1}{\mathbf{b}}-\varepsilon<\sum_{t_{1} \in \Psi_{m, n, t_{2}}^{-1}(k)} \frac{\boldsymbol{\sigma}_{m, n}^{(k)}\left(t_{1}, t_{2}\right)}{q_{m, n}}+\sum_{t_{1} \notin \Psi_{m, n, t_{2}}^{-1}(k)} \frac{\boldsymbol{\sigma}_{m, n}^{(k)}\left(t_{1}, t_{2}\right)}{q_{m, n}} \leq \frac{1}{\mathbf{b}}+\varepsilon, \\
& \frac{1}{\mathbf{b}}-d \varepsilon<\sum_{t_{1} \in \Psi_{m, n, t_{2}}^{-1}(k)} \frac{\boldsymbol{\sigma}_{m, n}^{(k)}\left(t_{1}, t_{2}\right)}{q_{m, n}} \leq \frac{1}{\mathbf{b}}+\varepsilon .
\end{aligned}
$$

We have proved that

$$
\sum_{t_{1} \in \Psi_{m, n, t_{2}}^{-1}(k)} \frac{\boldsymbol{\sigma}_{m, n}^{(k)}\left(t_{1}, t_{2}\right)}{q_{m, n}} \underset{m, n \rightarrow \infty}{\longrightarrow} \frac{1}{\mathbf{b}}
$$

uniformly in $m, n \in \mathbb{N}$ with $m<n$, which ends the proof.

From the proof of the previous lemma one can deduce that the values of $k_{m, n}\left(t_{1}, t_{2}\right)$ are ultimately uniquely defined if $\liminf _{m, n \rightarrow \infty, m<n} \frac{P_{m, n}\left(t_{1}, t_{2}\right)}{q_{m, n}}>0$. 


\section{Proof of Theorem 3}

In all this section $(X, T), \mu$ and $\left(q_{n} ; n \geq 1\right)$ are set as in Theorem 3 ,

5.1. Proof that the technical condition is necessary. It is enough to consider a non continuous eigenvalue $\lambda=\exp (2 i \pi / b)$ of $(X, T)$ for the ergodic measure $\mu$. Let $f \in L^{2}(X, \mu)$ be an associated eigenfunction with $|f|=1$.

Proof that the technical condition is necessary. Recall that $b /\left(b, p_{n}\right)$ is equal to $\mathbf{b}$ for all $n$ large enough. We know from Theorem 2 that $2 \leq \mathbf{b} \leq d$. Otherwise, if $\mathbf{b}=1$ the system would be linearly recurrent and $\lambda$ a continuous eigenvalue, as was discussed before stating Theorem 3. Thus we only need to prove statement (2) of the theorem.

It is enough to prove that for all $t_{1} \in\{1, \ldots, d\}$ and $t_{2} \in I_{\mu}$,

$$
\frac{P_{m, n}\left(t_{1}, t_{2}\right)}{q_{m, n}}-\frac{\left|\sum_{s \in \bar{S}_{m, n}\left(t_{1}, t_{2}\right)} \lambda^{-p_{m} s}\right|}{q_{m, n}} \underset{m, n \rightarrow \infty}{\longrightarrow} 0
$$

uniformly in $m, n \in \mathbb{N}$ with $m<n$. From here, we finish the proof adding over $t_{1} \in\{1, \ldots, d\}$.

First, we integrate $f$ over $B_{m}\left(t_{1}\right)$ and use the decomposition given in (2.7):

$$
\begin{aligned}
\int_{B_{m}\left(t_{1}\right)} f d \mu & =\sum_{t_{2} \in V_{n}} \sum_{s \in \bar{S}_{m, n}\left(t_{1}, t_{2}\right)} \int_{T^{-p_{m} s} B_{n}\left(t_{2}\right)} f d \mu \\
& =\sum_{t_{2} \in V_{n}} \sum_{s \in \bar{S}_{m, n}\left(t_{1}, t_{2}\right)} \int_{B_{n}\left(t_{2}\right)} f \circ T^{-p_{m} s} d \mu \\
& =\sum_{t_{2} \in V_{n}}\left(\sum_{s \in \bar{S}_{m, n}\left(t_{1}, t_{2}\right)} \lambda^{-p_{m} s}\right) \int_{B_{n}\left(t_{2}\right)} f d \mu .
\end{aligned}
$$

But, from (2.3), we have that

$$
\int_{B_{m}\left(t_{1}\right)} f d \mu=\mu_{m}\left(t_{1}\right) c_{m}\left(t_{1}\right) \lambda^{-\rho_{m}\left(t_{1}\right)}, \int_{B_{n}\left(t_{2}\right)} f d \mu=\mu_{n}\left(t_{2}\right) c_{n}\left(t_{2}\right) \lambda^{-\rho_{n}\left(t_{2}\right)} .
$$

Thus, substituting the corresponding expressions in the previous deduction we get

$$
\begin{gathered}
\mu_{m}\left(t_{1}\right) c_{m}\left(t_{1}\right) \lambda^{-\rho_{m}\left(t_{1}\right)}=\sum_{t_{2} \in V_{n}}\left(\sum_{s \in \bar{S}_{m, n}\left(t_{1}, t_{2}\right)} \lambda^{-p_{m} s}\right) \mu_{n}\left(t_{2}\right) c_{n}\left(t_{2}\right) \lambda^{-\rho_{n}\left(t_{2}\right)} \\
\mu\left(\tau_{m}=t_{1}\right) c_{m}\left(t_{1}\right) \lambda^{-\rho_{m}\left(t_{1}\right)}=\sum_{t_{2} \in V_{n}} \frac{\sum_{s \in \bar{S}_{m, n}\left(t_{1}, t_{2}\right)} \lambda^{-p_{m} s}}{q_{m, n}} \mu\left(\tau_{n}=t_{2}\right) c_{n}\left(t_{2}\right) \lambda^{-\rho_{n}\left(t_{2}\right)},
\end{gathered}
$$

where in the last equality we have used the relations $\mu\left(\tau_{m}=t_{1}\right)=p_{m} \mu_{m}\left(t_{1}\right)$, $\mu\left(\tau_{n}=t_{2}\right)=p_{n} \mu_{n}\left(t_{2}\right)$ and $p_{n} / p_{m}=q_{m, n}$. Using (4.2) we get the expression

$$
\mu\left(\tau_{m}=t_{1}\right) c_{m}\left(t_{1}\right) \lambda^{-\rho_{m}\left(t_{1}\right)}=\sum_{t_{2} \in V_{n}} \frac{\sigma_{m, n}\left(t_{1}, t_{2}\right)}{q_{m, n}} \mu\left(\tau_{n}=t_{2}\right) c_{n}\left(t_{2}\right) \lambda^{-\rho_{n}\left(t_{2}\right)} .
$$


From (2.2) we have that for $0<m<n$ and $t_{1} \in\{1, \ldots, d\}$

$$
\mu\left(\tau_{m}=t_{1}\right)=\sum_{t_{2} \in V_{n}} \frac{P_{m, n}\left(t_{1}, t_{2}\right)}{q_{m, n}} \mu\left(\tau_{n}=t_{2}\right) .
$$

Then, taking absolute value in (5.2) and using (4.6) and (5.3) we deduce

$$
\begin{aligned}
\mu\left(\tau_{m}=t_{1}\right) c_{m}\left(t_{1}\right) & \leq \sum_{t_{2} \in V_{n}} \frac{\left|\sigma_{m, n}\left(t_{1}, t_{2}\right)\right|}{q_{m, n}} \mu\left(\tau_{n}=t_{2}\right) c_{n}\left(t_{2}\right) \\
& \leq \sum_{t_{2} \in V_{n}} \frac{\left|\sigma_{m, n}\left(t_{1}, t_{2}\right)\right|}{q_{m, n}} \mu\left(\tau_{n}=t_{2}\right) \\
& \leq \sum_{t_{2} \in V_{n}} \frac{P_{m, n}\left(t_{1}, t_{2}\right)}{q_{m, n}} \mu\left(\tau_{n}=t_{2}\right) \\
& =\mu\left(\tau_{m}=t_{1}\right) .
\end{aligned}
$$

Notice that in the second inequality we have used that $c_{n}\left(t_{2}\right) \leq 1$ for any $n \in \mathbb{N}$ and $t_{2} \in V_{n}$.

Finally, applying Lemma 1 in the preceding inequalities we deduce that

$$
\sum_{t_{2} \in V_{n}}\left(\frac{P_{m, n}\left(t_{1}, t_{2}\right)}{q_{m, n}}-\frac{\left|\sigma_{m, n}\left(t_{1}, t_{2}\right)\right|}{q_{m, n}}\right) \mu\left(\tau_{n}=t_{2}\right) \underset{m, n \rightarrow \infty}{\longrightarrow} 0
$$

uniformly in $m, n \in \mathbb{N}$ with $m<n$. If $t_{2} \in I_{\mu}$, then $\mu\left(\tau_{n}=t_{2}\right)>\delta$ (recall $\delta$ comes from the cleanliness property of the diagram). Therefore, the desired convergence in (5.1) holds.

5.2. Proof that the technical condition is sufficient. For this proof we will need the following result from [BDM05] that we adapt to the language of BratteliVershik systems.

Theorem 14. Let $(X, T)$ be a minimal Cantor system given by a proper BratteliVershik system. A complex number $\lambda$ is an eigenvalue of $(X, T)$ with respect to the ergodic probability measure $\mu$ if and only if there exists a sequence of real functions $\rho_{n}: V_{n} \rightarrow \mathbb{R}, n \in \mathbb{N}$, such that

$$
\lambda^{r_{n}(x)+\rho_{n}\left(\tau_{n}(x)\right)} \text { converges }
$$

for $\mu$-almost every $x \in X$ when $n$ tends to infinity.

We recall that $r_{n}(x)=\bar{s}_{0}(x)+\sum_{i=1}^{n-1} p_{i} \bar{s}_{i}(x)$ is the entrance time of $x$ to $B_{n}\left(\tau_{n}(x)\right)$ (see (2.4)).

Proof that the technical condition is sufficient. We notice that condition (2) in Theorem 3 is stable under telescoping, so we will telescope our Bratteli-Vershik representation freely.

5.2.1. Constructing a partition. Take $t_{2} \in I_{\mu}$ and $m, n \in \mathbb{N}$ with $m<n$ enough large. Notice that our hypothesis is condition (1) in Lemma 12 with $t_{2} \in I_{\mu}$. Thus, for any $t_{1} \in\{1, \ldots, d\}$ there exist $k_{m, n}\left(t_{1}, t_{2}\right)$ given by condition (3) of Lemma 12 and the map $\Psi_{m, n, t_{2}}:\{1, \ldots, d\} \rightarrow\{0, \ldots, \mathbf{b}-1\}$ given by Lemma 13 , Define

$$
\mathcal{H}_{m, n, t_{2}}=\left\{A_{m, n, t_{2}}^{(0)}, A_{m, n, t_{2}}^{(1)}, \ldots, A_{m, n, t_{2}}^{(\mathbf{b}-1)}\right\},
$$

where $A_{m, n, t_{2}}^{(k)}=\Psi_{m, n, t_{2}}^{-1}(k)$ for $k \in\{0, \ldots, \mathbf{b}-1\}$. 
From Lemma 10 we can suppose after telescoping that $\mathcal{H}_{m, n, t_{2}}=\mathcal{H}_{m, m+1, t_{2}}$ for all $m, n \in \mathbb{N}$ with $m<n$ and $t_{2} \in I_{\mu}$. Thus we set $\mathcal{H}_{m, n, t_{2}}=\mathcal{H}_{m, t_{2}}$ and $A_{m, n, t_{2}}^{(k)}=$ $A_{m, t_{2}}^{(k)}$ for $k \in\{0, \ldots, \mathbf{b}-1\}$. In addition, after another telescoping, we can suppose $A_{m, t_{2}}^{(k)}=A_{m^{\prime}, t_{2}}^{(k)}$ for all $m, m^{\prime} \geq 1$ and $k \in\{0, \ldots, \mathbf{b}-1\}$. We set $\mathcal{H}_{t_{2}}=\mathcal{H}_{m, t_{2}}$, $A_{t_{2}}^{(k)}=A_{m, t_{2}}^{(k)}$ and thus $k\left(t_{1}, t_{2}\right)=k_{m, n}\left(t_{1}, t_{2}\right)$ for any $m, n \in \mathbb{N}$ with $m<n$, $t_{1} \in\{1, \ldots, d\}$ and $t_{2} \in I_{\mu}$.

5.2.2. Constructing a good set of full measure. For $m, n \in \mathbb{N}$ with $m<n$ consider the set

$$
\mathcal{C}_{m, n}=\left\{\tau_{n} \in I_{\mu}, \bar{s}_{m, n} \not \equiv_{\mathbf{b}} k\left(\tau_{m}, \tau_{n}\right)\right\} \cup\left\{\tau_{n} \notin I_{\mu}\right\} .
$$

Recall that the map $k\left(t_{1}, t_{2}\right)$ has been defined only for $t_{2} \in I_{\mu}$. Let us compute the measure of $\mathcal{C}_{m, n}$ :

$$
\begin{aligned}
& \mu\left(\mathcal{C}_{m, n}\right) \\
& =\sum_{t_{2} \in I_{\mu}} \sum_{t_{1} \in V_{m}} \mu\left(\tau_{m}=t_{1}, \tau_{n}=t_{2}, \bar{s}_{m, n} \not_{\mathbf{b}} k\left(t_{1}, t_{2}\right)\right)+\mu\left(\tau_{n} \notin I_{\mu}\right) \\
& =\sum_{t_{2} \in I_{\mu}} \sum_{t_{1} \in V_{m}}\left(P_{m, n}\left(t_{1}, t_{2}\right)-\boldsymbol{\sigma}_{m, n}^{\left(k\left(t_{1}, t_{2}\right)\right)}\left(t_{1}, t_{2}\right)\right) p_{m} \mu_{n}\left(t_{2}\right)+\mu\left(\tau_{n} \notin I_{\mu}\right) \\
& =\sum_{t_{2} \in I_{\mu}}\left(\sum_{t_{1} \in V_{m}} \frac{P_{m, n}\left(t_{1}, t_{2}\right)}{q_{m, n}}-\frac{\boldsymbol{\sigma}_{m, n}^{\left(k\left(t_{1}, t_{2}\right)\right)}\left(t_{1}, t_{2}\right)}{q_{m, n}}\right) \mu\left(\tau_{n}=t_{2}\right)+\mu\left(\tau_{n} \notin I_{\mu}\right),
\end{aligned}
$$

where we have used that $q_{m, n} p_{m}=p_{n}$ and $\mu\left(\tau_{n}=t_{2}\right)=p_{n} \mu_{n}\left(t_{2}\right)$.

Since condition (3) of Lemma 12 holds for $t_{2} \in I_{\mu}$ and $\mu\left(\tau_{n} \notin I_{\mu}\right)$ goes to 0 when $n$ tends to $\infty$ (recall the diagram is clean), then $\mu\left(\mathcal{C}_{m, n}\right) \underset{m, n \rightarrow \infty}{\longrightarrow} 0$ uniformly in $m, n \in \mathbb{N}$ with $m<n$.

Thus, we can telescope the diagram in order that

$$
\sum_{n \in \mathbb{N}} \mu\left(\mathcal{C}_{n, n+1}\right) \text { converges. }
$$

Hence, from the Borel-Cantelli Lemma we deduce that $\mu(\mathcal{C})=1$, where

$$
\mathcal{C}=\liminf _{n \rightarrow \infty} \mathcal{C}_{n, n+1}^{c}=\cup_{N \in \mathbb{N}} \cap_{n \geq N}\left\{\tau_{n} \in I_{\mu}, \bar{s}_{n} \equiv_{\mathbf{b}} k\left(\tau_{n}, \tau_{n+1}\right)\right\} .
$$

5.2.3. Constructing an eigenfunction. After telescoping we can suppose that $p_{n} \equiv_{b}$ $p$ for some $p \in\{0, \ldots, b-1\}$ and for all $n \geq 1$. This will transform expressions of the form $\lambda^{-p_{n} s}$ below to $\lambda^{-p s}$, which is independent of $n$.

For $m, n \in \mathbb{N}$ with $m<n, t_{1} \in\{1, \ldots, d\}$ and $t_{2} \in I_{\mu}$ we have

$$
\begin{aligned}
\sum_{s \in \bar{S}_{m, n}\left(t_{1}, t_{2}\right)} \lambda^{-p_{m} s} & =\sum_{\substack{s \in \bar{S}_{m, n}\left(t_{1}, t_{2}\right) \\
s \equiv_{\mathbf{b}} k\left(t_{1}, t_{2}\right)}} \lambda^{-p k\left(t_{1}, t_{2}\right)}+\sum_{\substack{s \in \bar{S}_{m, n}\left(t_{1}, t_{2}\right) \\
s \neq \mathbf{b} k\left(t_{1}, t_{2}\right)}} \lambda^{-p s} \\
& =P_{m, n}\left(t_{1}, t_{2}\right) \lambda^{-p k\left(t_{1}, t_{2}\right)}+\sum_{\substack{s \in \bar{S}_{m, n}\left(t_{1}, t_{2}\right) \\
s \neq \mathbf{b} k\left(t_{1}, t_{2}\right)}}\left(\lambda^{-p s}-\lambda^{-p k\left(t_{1}, t_{2}\right)}\right),
\end{aligned}
$$


where we have used that $\# \bar{S}_{m, n}\left(t_{1}, t_{2}\right)=P_{m, n}\left(t_{1}, t_{2}\right)$. Also, since

$$
\#\left\{s \in \bar{S}_{m, n}\left(t_{1}, t_{2}\right) ; s \not_{\mathbf{b}} k\left(t_{1}, t_{2}\right)\right\}=P_{m, n}\left(t_{1}, t_{2}\right)-\boldsymbol{\sigma}_{m, n}^{\left(k\left(t_{1}, t_{2}\right)\right)}\left(t_{1}, t_{2}\right),
$$

we have that

$$
\left|\sum_{\substack{s \in \bar{S}_{m, n}\left(t_{1}, t_{2}\right) \\ s \neq \mathbf{b} k\left(t_{1}, t_{2}\right)}}\left(\lambda^{-p s}-\lambda^{-p k\left(t_{1}, t_{2}\right)}\right)\right| \leq 2 \cdot\left(P_{m, n}\left(t_{1}, t_{2}\right)-\boldsymbol{\sigma}_{m, n}^{\left(k\left(t_{1}, t_{2}\right)\right)}\left(t_{1}, t_{2}\right)\right)
$$

As mentioned before, condition (2) of the main theorem using $t_{2} \in I_{\mu}$ implies that the equivalent conditions in Lemma 12 hold. So, by Lemma 12 (3), for $t_{1} \in$ $\{1, \ldots, d\}$ and $t_{2} \in I_{\mu}$ we have

$$
\frac{P_{m, n}\left(t_{1}, t_{2}\right)-\boldsymbol{\sigma}_{m, n}^{\left(k\left(t_{1}, t_{2}\right)\right)}\left(t_{1}, t_{2}\right)}{q_{m, n}} \underset{m, n \rightarrow \infty}{\longrightarrow} 0
$$

uniformly in $m, n \in \mathbb{N}$ with $m<n$.

We summarise previous discussion. Fix a real number $\epsilon>0$. Then, for all large enough $m, n \in \mathbb{N}$ with $m<n, t_{1} \in\{1, \ldots, d\}$ and $t_{2} \in I_{\mu}$ we can write

$$
\frac{1}{q_{m, n}} \sum_{s \in \bar{S}_{m, n}\left(t_{1}, t_{2}\right)} \lambda^{-p_{m} s}=\frac{P_{m, n}\left(t_{1}, t_{2}\right)}{q_{m, n}} \lambda^{-p k\left(t_{1}, t_{2}\right)}+\epsilon_{m, n}\left(t_{1}, t_{2}\right),
$$

where $\epsilon_{m, n}\left(t_{1}, t_{2}\right)$ is a complex number with $\left|\epsilon_{m, n}\left(t_{1}, t_{2}\right)\right| \leq \epsilon$.

Now, consider $\ell, m, n \in \mathbb{N}$ with $\ell<m<n$ enough large (such that the different uses of (5.6) below are valid), $t_{1} \in\{1, \ldots, d\}$ and $t_{3} \in I_{\mu}$. Then, by using (2.6) to get the second equality and (5.6) three times, we get

$$
\begin{aligned}
& \frac{P_{\ell, n}\left(t_{1}, t_{3}\right)}{q_{\ell, n}} \lambda^{-p k\left(t_{1}, t_{3}\right)}+\epsilon_{\ell, n}\left(t_{1}, t_{3}\right) \\
= & \frac{1}{q_{\ell, n}} \sum_{s \in \bar{S}_{\ell, n}\left(t_{1}, t_{3}\right)} \lambda^{-p_{\ell} s} \\
= & \frac{1}{q_{\ell, n}} \sum_{t_{2} \in V_{m}} \sum_{s_{1} \in \bar{S}_{l, m}\left(t_{1}, t_{2}\right)} \sum_{s_{2} \in \bar{S}_{m, n}\left(t_{2}, t_{3}\right)} \lambda^{-p_{\ell} s_{1}-p_{m} s_{2}} \\
= & \sum_{t_{2} \in V_{m}}\left(\frac{1}{q_{\ell, m}} \sum_{s_{1} \in \bar{S}_{\ell, m}\left(t_{1}, t_{2}\right)} \lambda^{-p_{\ell} s_{1}}\right)\left(\frac{1}{q_{m, n}} \sum_{s_{2} \in \bar{S}_{m, n}\left(t_{2}, t_{3}\right)} \lambda^{-p_{m} s_{2}}\right) \\
= & \sum_{t_{2} \in I_{\mu}}\left(\frac{P_{\ell, m}\left(t_{1}, t_{2}\right)}{q_{\ell, m}} \lambda^{-p k\left(t_{1}, t_{2}\right)}+\epsilon_{\ell, m}\left(t_{1}, t_{2}\right)\right)\left(\frac{P_{m, n}\left(t_{2}, t_{3}\right)}{q_{m, n}} \lambda^{-p k\left(t_{2}, t_{3}\right)}+\epsilon_{m, n}\left(t_{2}, t_{3}\right)\right) \\
& +\sum_{t_{2} \in V_{m} \backslash I_{\mu}}\left(\frac{1}{q_{\ell, m}} \sum_{s_{1} \in \bar{S}_{\ell, m}\left(t_{1}, t_{2}\right)} \lambda^{-p_{\ell} s_{1}}\right)\left(\frac{P_{m, n}\left(t_{2}, t_{3}\right)}{q_{m, n}} \lambda^{-p k\left(t_{2}, t_{3}\right)}+\epsilon_{m, n}\left(t_{2}, t_{3}\right)\right)
\end{aligned}
$$

Set $k\left(t_{1}, t_{2}\right)=0$ for $t_{1} \in\{1, \ldots, d\}$ and $t_{2} \notin I_{\mu}$ (recall that this map is only defined for $\left.t_{2} \in I_{\mu}\right)$. Adding and subtracting the terms $\frac{P_{\ell, m}\left(t_{1}, t_{2}\right)}{q_{\ell, m}} \lambda^{-p k\left(t_{1}, t_{2}\right)}$ when $t_{2} \notin I_{\mu}$ in the last equality of previous deduction gives 


$$
\begin{aligned}
& \frac{P_{\ell, n}\left(t_{1}, t_{3}\right)}{q_{\ell, n}} \lambda^{-p k\left(t_{1}, t_{3}\right)}+\epsilon_{\ell, n}\left(t_{1}, t_{3}\right) \\
& =\sum_{t_{2} \in I_{\mu}}\left(\frac{P_{\ell, m}\left(t_{1}, t_{2}\right)}{q_{\ell, m}} \lambda^{-p k\left(t_{1}, t_{2}\right)}+\epsilon_{\ell, m}\left(t_{1}, t_{2}\right)\right) \\
& +\sum_{t_{2} \in V_{m} \backslash I_{\mu}}\left(\frac{P_{\ell, m}\left(t_{1}, t_{2}\right)}{q_{\ell, m}} \lambda^{-p k\left(t_{1}, t_{2}\right)}\right)\left(\frac{P_{m, n}\left(t_{2}, t_{3}\right)}{q_{m, n}} \lambda^{-p k\left(t_{2}, t_{3}\right)}+\epsilon_{m, n}\left(t_{2}, t_{3}\right)\right) \\
& +\sum_{t_{2} \in V_{m} \backslash I_{\mu}}\left(\frac{1}{q_{\ell, m}} \sum_{s_{1} \in \bar{S}_{\ell, m}\left(t_{1}, t_{2}\right)} \lambda^{-p_{\ell} s_{1}}-\frac{P_{\ell, m}\left(t_{1}, t_{2}\right)}{q_{\ell, m}} \lambda^{-p k\left(t_{1}, t_{2}\right)}\right) \\
& \cdot\left(\frac{P_{m, n}\left(t_{2}, t_{3}\right)}{q_{m, n}} \lambda^{-p k\left(t_{2}, t_{3}\right)}+\epsilon_{m, n}\left(t_{2}, t_{3}\right)\right) .
\end{aligned}
$$

Finally, multiplying the terms we get that

$$
\begin{aligned}
\frac{P_{\ell, n}\left(t_{1}, t_{3}\right)}{q_{\ell, n}} \lambda^{-p k\left(t_{1}, t_{3}\right)} & +\epsilon_{\ell, n}\left(t_{1}, t_{3}\right) \\
& =\epsilon^{\prime}+\sum_{t_{2} \in V_{m}} \frac{P_{\ell, m}\left(t_{1}, t_{2}\right) P_{m, n}\left(t_{2}, t_{3}\right)}{q_{\ell, n}} \lambda^{-p\left(k\left(t_{1}, t_{2}\right)+k\left(t_{2}, t_{3}\right)\right)},
\end{aligned}
$$

where

$$
\left|\epsilon^{\prime}\right| \leq 2 d \epsilon+d \epsilon^{2}+d \epsilon+\sum_{t_{2} \in V_{m} \backslash I_{\mu}} 2 \cdot \frac{P_{m, n}\left(t_{2}, t_{3}\right)}{q_{m, n}}+2 d \epsilon .
$$

But, for $t_{2} \notin I_{\mu}, t_{3} \in I_{\mu}$ and any large $m, n \in \mathbb{N}$ with $m<n$ we have that $\mu\left(\tau_{n}=t_{3}\right) \geq \delta$ and $\mu\left(\tau_{m}=t_{2}\right) \leq \delta \epsilon$, where $\delta$ comes from the definition of a clean Bratteli-Vershik representation. Consequently, using equality (5.3), we have that

$$
\frac{P_{m, n}\left(t_{2}, t_{3}\right)}{q_{m, n}} \leq \frac{\mu\left(\tau_{m}=t_{2}\right)}{\mu\left(\tau_{n}=t_{3}\right)} \leq \frac{\mu\left(\tau_{m}=t_{2}\right)}{\delta} \leq \epsilon .
$$

Thus, combining (5.9) in (5.8), we get

$$
\left|\epsilon^{\prime}\right| \leq 5 d \epsilon+2 d \epsilon \leq 8 d \epsilon .
$$

Now, a simple reordering of terms in (5.7) gives

$$
\begin{aligned}
1+\frac{q_{\ell, n}}{P_{\ell, n}\left(t_{1}, t_{3}\right)} & \left(\epsilon_{\ell, n}\left(t_{1}, t_{3}\right)-\epsilon^{\prime}\right) \lambda^{p k\left(t_{1}, t_{3}\right)} \\
& =\sum_{t_{2} \in V_{m}} \frac{P_{\ell, m}\left(t_{1}, t_{2}\right) P_{m, n}\left(t_{2}, t_{3}\right)}{P_{\ell, n}\left(t_{1}, t_{3}\right)} \lambda^{p\left(k\left(t_{1}, t_{3}\right)-k\left(t_{1}, t_{2}\right)-k\left(t_{2}, t_{3}\right)\right)} .
\end{aligned}
$$


Recall from Lemma 11 that for every $\ell \in \mathbb{N}$ enough large there exist integers $m, n$ with $n>m>\ell$ such that for all $t_{1}, t_{2}, t_{3} \in I_{\mu}$,

$$
\frac{P_{\ell, n}\left(t_{1}, t_{3}\right)}{q_{\ell, n}} \geq \frac{\delta}{3}, \frac{P_{\ell, m}\left(t_{1}, t_{2}\right)}{q_{\ell, m}} \geq \frac{\delta}{3} \text { and } \frac{P_{m, n}\left(t_{2}, t_{3}\right)}{q_{m, n}} \geq \frac{\delta}{3} .
$$

Then, if considering $t_{1}, t_{3} \in I_{\mu}$ and fixing integers $\ell, m, n \in \mathbb{N}$ with $\ell<m<n$ enough large to verify (5.12), and using (5.10), we get

$$
1+\epsilon^{\prime \prime}=\sum_{t_{2} \in V_{m}} \frac{P_{\ell, m}\left(t_{1}, t_{2}\right) P_{m, n}\left(t_{2}, t_{3}\right)}{P_{\ell, n}\left(t_{1}, t_{3}\right)} \lambda^{p\left(k\left(t_{1}, t_{3}\right)-k\left(t_{1}, t_{2}\right)-k\left(t_{2}, t_{3}\right)\right)},
$$

where $\left|\epsilon^{\prime \prime}\right| \leq \hat{C} \epsilon$ and $\hat{C}$ is a positive constant only depending on the system.

Let us show that $p k\left(t_{1}, t_{3}\right) \equiv_{b} p\left(k\left(t_{1}, t_{2}\right)+k\left(t_{2}, t_{3}\right)\right)$ for all $t_{2} \in I_{\mu}$. We rewrite the right hand side of (5.13), which is a convex sum, as $\sum_{i=0}^{b-1} \alpha_{i} \lambda^{i}$, where

$$
\alpha_{i}=\sum_{\left\{t_{2} \in V_{m} ; p\left(k\left(t_{1}, t_{3}\right)-k\left(t_{1}, t_{2}\right)-k\left(t_{2}, t_{3}\right)\right) \equiv_{b} i\right\}} \frac{P_{\ell, m}\left(t_{1}, t_{2}\right) P_{m, n}\left(t_{2}, t_{3}\right)}{P_{\ell, n}\left(t_{1}, t_{3}\right)} .
$$

By (5.13), we can use Lemma 9. Then, there is $i_{0} \in\{0, \ldots, b-1\}$ such that $\alpha_{i_{0}}>1-C \hat{C} \epsilon$ ( $C$ is the constant of Lemma9 for the $b$-th roots of unity). Moreover, if $\epsilon$ was taken small enough, we have that $i_{0}=0$ since the convex combination is close to 1 . But, again using (5.12), for all $t_{2} \in I_{\mu}$,

$$
\frac{P_{\ell, m}\left(t_{1}, t_{2}\right) P_{m, n}\left(t_{2}, t_{3}\right)}{P_{\ell, n}\left(t_{1}, t_{3}\right)}=\frac{P_{\ell, m}\left(t_{1}, t_{2}\right)}{q_{\ell, m}} \frac{P_{m, n}\left(t_{2}, t_{3}\right)}{q_{m, n}} \frac{q_{\ell, n}}{P_{\ell, n}\left(t_{1}, t_{3}\right)} \geq \frac{\delta^{2}}{9}>C \hat{C} \epsilon
$$

if $\epsilon$ was taken small enough. Since $\alpha_{i_{0}}>1-C \hat{C} \epsilon$, then for all $t_{2} \in I_{\mu}$,

$$
p\left(k\left(t_{1}, t_{3}\right)-k\left(t_{1}, t_{2}\right)-k\left(t_{2}, t_{3}\right)\right) \equiv_{b} i_{0}=0 .
$$

This proves our claim.

Summarising, we have proved that for all $t_{1}, t_{2}, t_{3} \in I_{\mu}$,

$$
\begin{gathered}
p \cdot k\left(t_{1}, t_{3}\right) \equiv_{b} p \cdot k\left(t_{1}, t_{2}\right)+p \cdot k\left(t_{2}, t_{3}\right), \\
p \cdot k\left(t_{1}, t_{1}\right) \equiv_{b} 0, \quad p \cdot k\left(t_{1}, t_{2}\right) \equiv_{b}-p \cdot k\left(t_{2}, t_{1}\right) .
\end{gathered}
$$

To finish we will verify the criterium of Theorem 14 for $\lambda=\exp (2 i \pi / b)$. Fix an element $t_{0} \in I_{\mu}$ and for each $n \geq 1$ define $\rho_{n}: V_{n} \rightarrow \mathbb{R}$ by $\rho_{n}(t)=-p k\left(t_{0}, t\right)$.

Let $x$ be an element in $\mathcal{C}$. By definition of $\mathcal{C}$, there exists $N \in \mathbb{N}$ such that for any $n \geq N, \tau_{n}(x) \in I_{\mu}$ and $\bar{s}_{n}(x) \equiv_{\mathbf{b}} k\left(\tau_{n}(x), \tau_{n+1}(x)\right)$. Notice that, since $p \mathbf{b}$ is divisible by $b$ (recall that $\mathbf{b}=p /(b, p)$ ), then after multiplying by $p$ we get that $p \bar{s}_{n}(x) \equiv_{b} p k\left(\tau_{n}(x), \tau_{n+1}(x)\right)$. Then, for $n \geq N$ one has,

$$
\begin{aligned}
\left|\lambda^{r_{n+1}(x)+\rho_{n+1}\left(\tau_{n+1}(x)\right)}-\lambda^{r_{n}(x)+\rho_{n}\left(\tau_{n}(x)\right)}\right| & =\left|\lambda^{r_{n+1}(x)-r_{n}(x)+\rho_{n+1}\left(\tau_{n+1}(x)\right)-\rho_{n}\left(\tau_{n}(x)\right)}-1\right| \\
& =\left|\lambda^{p \bar{s}_{n}(x)-p k\left(t_{0}, \tau_{n+1}(x)\right)+p k\left(t_{0}, \tau_{n}(x)\right)}-1\right| \\
& =\left|\lambda^{p \bar{s}_{n}(x)-\left(p k\left(\tau_{n}(x), t_{0}\right)+p k\left(t_{0}, \tau_{n+1}(x)\right)\right)}-1\right| \\
& =\left|\lambda^{p \bar{s}_{n}(x)-p k\left(\tau_{n}(x), \tau_{n+1}(x)\right)}-1\right|=0,
\end{aligned}
$$


where to deduce the second equality we have used (2.4) and to derive the last one we applied (5.14) and (5.15). This proves that $\lambda^{r_{n}(x)+\rho_{n}\left(\tau_{n}(x)\right)}$ is eventually constant, so it converges. We finish the proof using Theorem 14 .

Let us remark that from the previous proof Corollary 4 follows directly. In fact, it is just a reformulation of the last part of the proof.

5.3. Proof of Corollary 7. (1) Let $\mu$ be an ergodic measure such that $\mathbf{B}_{\mu}$ is non empty. Let $\lambda=\exp (2 i \pi a / b)$ be a non continuous eigenvalue for $\mu$ such that $b /\left(b, p_{n}\right)=\mathbf{b} \in \mathbf{B}_{\mu}$ for all large enough integers $n \in \mathbb{N}$. The hypotheses of Lemma 13 hold for all $t_{2} \in I_{\mu}$ using this value of $\lambda$. Then, from Lemma 13 (2), for every $t_{2} \in I_{\mu}$ and $k \in\{0, \ldots, \mathbf{b}-1\}$ the sum

$$
\begin{aligned}
& \sum_{t_{1} \in \Psi_{m, n, t_{2}}^{-1}(k)} \frac{\boldsymbol{\sigma}_{m, n}^{(k)}\left(t_{1}, t_{2}\right)}{q_{m, n}} \\
= & \sum_{t_{1} \in \Psi_{m, n, t_{2}}^{-1}(k) \cap I_{\mu}} \frac{\boldsymbol{\sigma}_{m, n}^{(k)}\left(t_{1}, t_{2}\right)}{q_{m, n}}+\sum_{t_{1} \in \Psi_{m, n, t_{2}}^{-1}(k) \cap I_{\mu}^{c}} \frac{\boldsymbol{\sigma}_{m, n}^{(k)}\left(t_{1}, t_{2}\right)}{q_{m, n}}
\end{aligned}
$$

converges uniformly in $m, n \in \mathbb{N}$ with $m<n$ to $1 / \mathbf{b}$. But, since $\frac{\boldsymbol{\sigma}_{m, n}^{(k)}\left(t_{1}, t_{2}\right)}{q_{m, n}} \leq$ $\frac{P_{m, n}\left(t_{1}, t_{2}\right)}{q_{m, n}}$, from (3.1) we deduce that

$$
\sum_{t_{1} \in \Psi_{m, n, t_{2}}^{-1}(k) \cap I_{\mu}} \frac{\boldsymbol{\sigma}_{m, n}^{(k)}\left(t_{1}, t_{2}\right)}{q_{m, n}} \underset{m, n \rightarrow \infty}{\longrightarrow} \frac{1}{\mathbf{b}}, \sum_{t_{1} \in \Psi_{m, n, t_{2}}^{-1}(k) \cap I_{\mu}^{c}} \frac{\boldsymbol{\sigma}_{m, n}^{(k)}\left(t_{1}, t_{2}\right)}{q_{m, n}} \underset{m, n \rightarrow \infty}{\longrightarrow} 0,
$$

converges uniformly in $m, n \in \mathbb{N}$ with $m<n$.

We deduce that for any large enough $m, n \in \mathbb{N}$ with $m<n, t_{2} \in I_{\mu}$ and $k \in$ $\{0, \ldots, \mathbf{b}-1\}$ each set $\Psi_{m, n, t_{2}}^{-1}(k)$ must contain an element of $I_{\mu}$. Thus $\# I_{\mu} \geq \mathbf{b}$.

(2) Let us consider $\mu \in \mathcal{M}_{\text {erg }}(X, T)$ such that $\mathbf{B}_{\mu} \neq \emptyset$. Let $\exp \left(2 i \pi / b_{1}\right)$ and $\exp \left(2 i \pi / b_{2}\right)$ be two different non continuous eigenvalues for $\mu$. Then, by Bezout's identity, $\exp \left(2 i \pi / \operatorname{lcm}\left(b_{1}, b_{2}\right)\right)$ is also an eigenvalue for $\mu$. Moreover, it is a non continuous eigenvalue. Indeed, if this fact is not true, then for some $n \in \mathbb{N}$ and $a \in \mathbb{Z}$ we have that $1 / \operatorname{lcm}\left(b_{1}, b_{2}\right)=a / p_{n}$. This implies that $1 / b_{1}=\left(a \operatorname{lcm}\left(b_{1}, b_{2}\right) / b_{1}\right) / p_{n}$ which is a contradiction since $\exp \left(2 i \pi / b_{1}\right)$ is a non continuous eigenvalue. This proves our claim.

Denote $\operatorname{lcm}\left(b_{1}, b_{2}\right)$ by $b$. Decomposing $b_{1}=\mathfrak{b}_{1} \cdot \mathfrak{b}_{2} \cdot \mathfrak{b}_{3} \cdot \mathfrak{b}_{4}$ and $b_{2}=\mathfrak{b}_{3} \cdot \mathfrak{b}_{4} \cdot \mathfrak{b}_{5} \cdot \mathfrak{b}_{6}$, where $\left(b_{1}, b_{2}\right)=\mathfrak{b}_{3} \cdot \mathfrak{b}_{4},\left(b_{1}, p_{n}\right)=\mathfrak{b}_{2} \cdot \mathfrak{b}_{3}$ and $\left(b_{2}, p_{n}\right)=\mathfrak{b}_{3} \cdot \mathfrak{b}_{5}$, we get the identity

$$
\operatorname{lcm}\left(\frac{b_{1}}{\left(b_{1}, p_{n}\right)}, \frac{b_{2}}{\left(b_{2}, p_{n}\right)}\right)=\frac{b}{\left(b, p_{n}\right)} \text {. }
$$

From this identity follows that it is not possible to have more than one divisibilitymaximal element in $\mathbf{B}_{\mu}$.

(3) For different ergodic measures $\mu$ and $\nu$ we have $I_{\mu} \cap I_{\nu}=\emptyset$ (recall that the Bratteli-Vershik representation is clean). Then,

$$
\sum_{\mu \in \mathcal{M}_{e r g}(X, T)} \# I_{\mu} \leq d .
$$

But, (1) implies that $\mathbf{b}_{\mu} \leq \# I_{\mu}$ for each $\mu \in \mathcal{M}$, so (3) follows. 
(4) As in the proof of (3), we use that for different ergodic measures $\mu$ and $\nu$ we have $I_{\mu} \cap I_{\nu}=\emptyset$. Hence,

$$
\begin{aligned}
\# \mathcal{M} \leq \# \mathcal{M}_{\text {erg }}(X, T) & =\sum_{\mu \in \mathcal{M}_{\text {erg }}(X, T)} \# I_{\mu}-\sum_{\mu \in \mathcal{M}_{\text {erg }}(X, T)}\left(\# I_{\mu}-1\right) \\
& \leq d-\sum_{\mu \in \mathcal{M}}\left(\mathbf{b}_{\mu}-1\right),
\end{aligned}
$$

where in the inequality we used (1). This proves (4).

5.4. Proof of Corollary 8. Consider $\lambda=\exp (2 i \pi / b)$ with $b$ an integer such that $b /\left(b, p_{n}\right)=d$ for all $n$ large enough.

First we prove the necessary and sufficient condition given by (3.2). If $\lambda$ is a non continuous eigenvalue, then $\mathbf{b}_{\mu}$ defined in Corollary $\mathbf{7}$ is equal to $d$. In addition, since $\mathbf{b}_{\mu}=d$, the partition of Corollary 6 is made of singletons and we get the property (3.2) for any $t_{2} \in I_{\mu}$. But, using statement (1) of Corollary 7 , one deduces that $I_{\mu}=\{1, \ldots, d\}$. Thus property (3.2) is true for any $t_{2} \in\{1, \ldots, d\}$. Clearly, property (3.2) implies that $\lambda$ is a non continuous eigenvalue by Corollary 6 , Now, assume $\lambda$ is a non continuous eigenvalue. Using Corollary 7 (3) one gets that $\mathcal{M}_{\text {erg }}(X, T)$ has a unique element, so the system is uniquely ergodic. This proves statement (1).

Finally we prove statement (2). Recall that under our hypothesis equivalent conditions of Lemma 12 hold for any $t_{2} \in\{1, \ldots, d\}$. Then, from the equality

$$
\left|\mu\left(\tau_{m}=t_{1}\right)-\frac{1}{d}\right|=\left|\sum_{t_{2} \in V_{n}}\left(\frac{P_{m, n}\left(t_{1}, t_{2}\right)}{q_{m, n}}-\frac{1}{d}\right) \mu\left(\tau_{n}=t_{2}\right)\right|,
$$

(3.2) and Lemma 12 (2) one gets

$$
\lim _{m \rightarrow \infty} \mu\left(\tau_{m}=t_{1}\right)=\frac{1}{d}
$$

This proves the desired statement.

\section{EXAMPLES}

6.1. Example 1: A model example. We start with a basic model example that will be used later to illustrate several behaviors of the eigenvalues with respect to the ergodic measures. We start with a general framework to construct a family of examples where the Bratteli-Vershik representations are not necessarily proper. Later we modify this family to obtain proper representations. Finally, we prove that in this family of examples all ergodic measures share the same non continuous eigenvalue $\exp (2 i \pi / 6)$.

6.1.1. Define the sequence $q_{1}=1, q_{2}=2 \cdot 5^{2}$ and $q_{n}=5^{2 n}$ for $n>2$. First, consider the (non necessarily proper) Toeplitz diagram with the characteristic sequence $\left(q_{n} ; n \in \mathbb{N}\right)$ such that $V_{n}=\{1,2,3,4,5,6,7\}$ for all $n \geq 1$ and the local order of the $q_{n+1}$ arrows arriving at $t \in V_{n+1}$ is given by the following associated sequences of vertices in $V_{n}$ :

$$
t \rightarrow v_{n+1}(t) \text { for all } 1 \leq t \leq 7,
$$

where each $v_{n+1}(t)$ is a fixed word of length $q_{n+1}$ on the alphabet $V_{n}$ built in the following way: 
(1) Set $W_{1}=\{1,4,7\}, W_{2}=\{2,5\}$ and $W_{3}=\{3,6\}$.

(2) For $n \geq 2$ the words $v_{n+1}(1), v_{n+1}(4)$ and $v_{n+1}(7)$ begin with an element of $W_{1}$, followed by an element of $W_{2}$ and this is followed by an element of $W_{3}$. Then we restart from $W_{1}$ and so on. Because $q_{n+1} \equiv_{3} 1$ for $n \geq 2$, all these three words end with an element of $W_{1}$. The words $v_{n+1}(2)$ and $v_{n+1}(5)$ follow the same periodic scheme starting with an element of $W_{2}$, then of $W_{3}$ and so on (and therefore ending with an element of $\left.W_{2}\right)$. And finally the words $v_{n+1}(3)$ and $v_{n+1}(6)$ follow the periodic scheme starting in $W_{3}$.

(3) Level 2 is built in any way.

Define $k:\{1, \ldots, 7\} \times\{1, \ldots, 7\} \rightarrow\{0,1,2\}$ by: $k\left(t_{1}, t_{2}\right)=j-i \bmod 3$ if $t_{1} \in W_{i}$ and $t_{2} \in W_{j}$. The following two properties are straightforward:

- For $t_{1}, t_{2}, t_{3} \in\{1, \ldots, 7\}$ we have

$$
k\left(t_{1}, t_{3}\right) \equiv_{3} k\left(t_{1}, t_{2}\right)+k\left(t_{2}, t_{3}\right) .
$$

- Let $x$ be an infinite sequence in the ordered Bratteli diagram. For $n \geq 2$,

$$
\bar{s}_{n}(x) \equiv_{3} k\left(\tau_{n}(x), \tau_{n+1}(x)\right) .
$$

6.1.2. Now we modify a little bit the previously defined local orders to get a proper Bratteli-Vershik representation for the system. To produce the new orders we change sequences $v_{n+1}(t)$ into $w_{n+1}(t)$ in such a way that: $(1) w_{n+1}(t)=v_{n+1}(t)$, except for at most a fixed number of letters, say $L$, independent of $n ;(2) w_{n+1}(t)$ begins and ends with 1 ; and (3) $w_{n+1}(t)$ contains every element of $\{1,2,3,4,5,6,7\}$ at least once. This diagram is clearly proper and induces a Toeplitz system of finite $\operatorname{rank}(X, T)$.

Consider any invariant measure $\mu$ on the system. We prove that $\exp (2 \pi i / 6)$ is a non continuous eigenvalue of $(X, T)$ for $\mu$, and this fact is independent of the measure $\mu$ we choose. In order to do that, we verify conditions (1) to (4) of Corollary 4 .

By construction $p_{n} \equiv_{6} 2$ and $\mathbf{b}=6 /\left(6, p_{n}\right)=3$ for all $n \geq 2$, so conditions (1) and (2) hold. Condition (3) follows directly from (6.1). To prove condition (4) we need to find a set of full measure where $\bar{s}_{n}(x) \equiv_{3} k\left(\tau_{n}(x), \tau_{n+1}(x)\right)$ for all large enough $n \in \mathbb{N}$.

Similarly as in the proof of Theorem 3, for $n \in \mathbb{N}$ consider $\mathcal{C}_{n}=\left\{x \in X ; \bar{s}_{n}(x) \not \equiv_{3}\right.$ $\left.k\left(\tau_{n}(x), \tau_{n+1}(x)\right)\right\}$. Since (6.2) holds before modifying the orders and those modifications alter no more than $L$ letters per level, we easily check that

$$
\begin{aligned}
\mu\left(\mathcal{C}_{n}\right) & =\sum_{t_{1}=1}^{7} \sum_{t_{2}=1}^{7} \mu\left(\tau_{n}=t_{1}, \tau_{n+1}=t_{2}, \bar{s}_{n}(x) \neq_{3} k\left(t_{1}, t_{2}\right)\right) \\
& \leq \sum_{t_{1}=1}^{7} \sum_{t_{2}=1}^{7} L h_{n}\left(t_{1}\right) \mu_{n+1}\left(t_{2}\right) \\
& \leq \sum_{t_{1}=1}^{7} \sum_{t_{2}=1}^{7} \frac{L}{q_{n+1}} \mu\left(\tau_{n+1}=t_{2}\right) \\
& \leq \frac{7 L}{q_{n+1}}
\end{aligned}
$$

So, $\sum_{n>1} \mu\left(\mathcal{C}_{n}\right)$ converges. Hence, from the Borel-Cantelli Lemma we get $\mu(\mathcal{C})=1$, where $\mathcal{C}=\liminf _{n \rightarrow \infty} \mathcal{C}_{n}^{c}$. 
6.2. Example 2: A first particular case of the model example. In this example we precise the construction of Example 1 in order to show that the model example can produce a uniquely ergodic system, where $\exp (2 i \pi / 6)$ is a non continuous eigenvalue for the unique invariant measure. In addition, this will illustrate that inequalities in Corollary 7 can be strict and that Corollary 8 is not reversible since one can have $\mathbf{b}_{\mu}<d$ in the uniquely ergodic case.

First, for $n \geq 3$ define $c_{n}$ such that $q_{n}=12 c_{n}+1$ and define words giving the order of the diagram by

$$
\begin{aligned}
& w_{n+1}(1)=(123456723756)^{c_{n+1}} 1 \\
& w_{n+1}(2)=1(312645372675)^{c_{n+1}-1}(312)^{3} 671 \\
& w_{n+1}(3)=1(123456723756)^{c_{n+1}-1}(123)^{3} 451 \\
& w_{n+1}(4)=(156423756723)^{c_{n+1} 1} \\
& w_{n+1}(5)=1(345612375672)^{c_{n+1}-1}(645)^{3} 311 \\
& w_{n+1}(6)=1(156423756723)^{c_{n+1}-1}(723)^{3} 121 \\
& w_{n+1}(7)=(153426753726)^{c_{n+1}} 1
\end{aligned}
$$

It is straightforward that these orders fit the model construction in Example 1. Also, for any invariant measure $\mu$, the system satisfies: $\mu\left(\tau_{n}=t\right) \underset{n \rightarrow \infty}{\longrightarrow} 1 / 6$ for $t=2,3,5,6,7$ and $\mu\left(\tau_{n}=t\right) \underset{n \rightarrow \infty}{\longrightarrow} 1 / 12$ for $t=1,4$. The proof is a simple computation. For example,

$$
\frac{c_{n+1}+1}{q_{n+1}}<\mu\left(\tau_{n}=1\right)<\frac{c_{n+1}+4}{q_{n+1}},
$$

and then we use that $c_{n+1} / q_{n+1} \underset{n \rightarrow \infty}{\longrightarrow} 1 / 12$. Since $\# I_{\mu}=7$, then we deduce that the system is uniquely ergodic. Also, since 3 divides $\mathbf{b}_{\mu}$, then $\mathbf{b}_{\mu}<\# I_{\mu}$.

6.3. Example 3: A second particular case of the model example. Here we will use the model example to produce a Bratteli-Vershik system having exactly two ergodic measures. Then, for each one $\exp (2 i \pi / 6)$ is a non continuous eigenvalue. Let us take in the model example the following particular choice of $w_{n+1}(t)$ for $t \in\{1, \ldots, 7\}$ and $n \geq 2$. First define $c_{n}$ so that $q_{n}=3 c_{n}+1$, and then set:

$$
\begin{aligned}
& w_{n+1}(1)=(123)^{c_{n+1}-2} 4567231 \\
& w_{n+1}(2)=13(123)^{c_{n+1}-2} 45671 \\
& w_{n+1}(3)=1(123)^{c_{n+1}-2} 456721 \\
& w_{n+1}(4)=146(456)^{c_{n+1}-2} 7231 \\
& w_{n+1}(5)=14(456)^{c_{n+1}-2} 12371 \\
& w_{n+1}(6)=1(456)^{c_{n+1}-2} 123761 \\
& w_{n+1}(7)=156(456)^{c_{n+1}-2} 7231
\end{aligned}
$$

As was shown in Theorem 3.3 (2) of [BKMS13, any ergodic measure is obtained as an extension of a finite measure on a system defined on a subdiagram. A subdiagram is obtained fixing subsets of vertices at each level and considering only the paths which go along the vertices in such subsets. The order is defined naturally following the order of the complete diagram. Here we will fix a unique subset of $\{1, \ldots, 7\}$ for all levels. 
Consider the subset $A_{1}=\{1,2,3\}$ and construct the associated subdiagram. Using the same nomenclature as before, for levels $n \geq 2$ the corresponding subdiagram has the following induced local orders:

$$
\begin{aligned}
& 1 \rightarrow(123)^{c_{n+1}-2} 231 \\
& 2 \rightarrow 13(123)^{c_{n+1}-2} 1 \\
& 3 \rightarrow 1(123)^{c_{n+1}-2} 21
\end{aligned}
$$

This order determines a proper diagram that is of Toeplitz type and has the characteristic sequence $\left(\bar{q}_{n}\right)_{n \in \mathbb{N}}$, with $\bar{q}_{n}=q_{n}-4$ for $n>2$. Analogously to Example 2 , we can see that the system $(Y, S)$ induced by this diagram is uniquely ergodic. Moreover, the unique invariant measure $\mu$ of this system can be naturally extended to a finite ergodic measure of $(X, T)$. For a deeper discussion of this extension we refer the reader to BKMS13 Section 3. Let us call $\widehat{\mu}$ the normalized extension of $\mu$. Then $\widehat{\mu}$ is an ergodic probability measure on $(X, T)$.

Analogously, consider $A_{2}=\{4,5,6\}$. In this case the corresponding subdiagram has the following local orders. For $n \geq 2$,

$$
\begin{aligned}
& 4 \rightarrow 46(456)^{c_{n+1}-2} \\
& 5 \rightarrow 4(456)^{c_{n+1}-2} \\
& 6 \rightarrow(456)^{c_{n+1}-2} 6
\end{aligned}
$$

This diagram has unique maximal and minimal paths, the words have lengths $q_{n+1}-5, q_{n+1}-6$ and $q_{n+1}-6$ respectively. As before, one proves that the system $(Z, R)$ associated to this diagram is uniquely ergodic and that the unique ergodic measure $\nu$ can be extended to a finite ergodic measure of $(X, T)$. We call $\widehat{\nu}$ the normalized extension of $\nu$.

From Theorem 3.3 (4) in BKMS13 one deduces that $(X, T)$ has no other ergodic probability measures than $\widehat{\mu}$ and $\widehat{\nu}$. Furthermore, one proves by simple computations that the diagram is clean and $I_{\widehat{\mu}}=\{1,2,3\}$ and $I_{\widehat{\nu}}=\{4,5,6\}$.

6.4. Example 4: A small variation of the model example. We provide an example of a finite rank Toeplitz system with two ergodic measures. For one there is a non continuous eigenvalue, while for the other all eigenvalues are continuous. We keep the values for $q_{n}$ of Example 1 but we consider the following choice of $w_{n+1}(t)$ for $t \in\{1, \ldots, 7\}$ and $n \geq 2$, where $c_{n}$ is such that $q_{n}=12 c_{n}+1$ :

$$
\begin{aligned}
& w_{n+1}(1)=(123456423156)^{c_{n+1}-1}(123)^{3} 7561 \\
& w_{n+1}(2)=1(312645342615)^{c_{n+1}-1}(312)^{3} 671 \\
& w_{n+1}(3)=1(123456423156)^{c_{n+1}-2}(123)^{3} 751 \\
& w_{n+1}(4)=(156423456123)^{c_{n+1}-1}(123)^{3} 7561 \\
& w_{n+1}(5)=1(345612315642)^{c_{n+1}-1}(645)^{3} 371 \\
& w_{n+1}(6)=1(156423456123)^{c_{n+1}-2}(123)^{3} 721 \\
& w_{n+1}(7)=1(7)^{q_{n+1}-7} 654321
\end{aligned}
$$

This order does not fit conditions of Example 1, so we cannot ensure that $\exp (2 i \pi / 6)$ is a non continuous eigenvalue for every ergodic measure $\mu$ on the system $(X, T)$ induced by this diagram. 
As in the previous example one proves that the subdiagrams associated to the subsets of vertices $\{1,2,3,4,5,6\}$ and $\{7\}$ at all levels define systems $(Y, S)$ and $(Z, R)$ respectively, which are uniquely ergodic and the normalized extensions of their unique probability measures, $\widehat{\mu}$ and $\widehat{\nu}$, are ergodic measures on $(X, T)$. Furthermore, a detailed computation allows to prove that the diagram is clean with respect to these measures and that $I_{\widehat{\mu}}=\{1,2,3,4,5,6\}$ and $I_{\widehat{\nu}}=\{7\}$. This implies there is no other ergodic probability measure on $(X, T)$ aside from such extensions. Now we prove that $\exp (2 i \pi / 6)$ is a non continuous eigenvalue for $\widehat{\mu}$ and that $\widehat{\nu}$ does not have non continuous eigenvalues. The only difference between the model example and this case is the measure of the set

$$
\mathcal{C}_{n}=\left\{x \in X ; \bar{s}_{n}(x) \not \equiv_{3} k\left(\tau_{n}(x), \tau_{n+1}(x)\right)\right\} .
$$

Here, $\widehat{\mu}\left(\mathcal{C}_{n}\right) \leq \frac{2}{q_{n+1}}+\widehat{\mu}\left(\tau_{n+1}=7\right)$ and a simple computation allows to prove that $\sum_{n \geq 1} \widehat{\mu}\left(\mathcal{C}_{n}\right)$ converges. We deduce by using Corollary 4 that $\exp (2 i \pi / 6)$ is a non continuous eigenvalue for $\widehat{\mu}$.

The absence of non continuous rational eigenvalues, say $\lambda=\exp (2 i \pi / b)$, for $\widehat{\nu}$ follows from inequalities $1<b /\left(b, p_{n}\right) \leq \# I_{\widehat{\nu}}=1$, which is a contradiction.

6.5. Example 5: A big variation of the model example. Here we provide a Bratteli-Vershik system of Toeplitz type with rank 7 having two ergodic measures and different non continuous eigenvalues associated to them. The first eigenvalue is $\exp (2 i \pi / 6)$ and the corresponding $\mathbf{b}=3$, and the other eigenvalue is $\exp (2 i \pi / 8)$ with $\mathbf{b}=4$. In particular, this example shows that all inequalities of Corollary 7 (4) can be equalities. We keep the values for $q_{n}$ of Example 1 and for $t \in\{1, \ldots, 7\}$ and $n \geq 2$ we consider the following choice of $w_{n+1}(t)$, where $c_{n}$ is such that $q_{n}=12 c_{n}+1$ :

$$
\begin{aligned}
& w_{n+1}(1)=(123)^{4 c_{n+1}-2} 1245671 \\
& w_{n+1}(2)=1(312)^{4 c_{n+1}-2} 345671 \\
& w_{n+1}(3)=1(123)^{4 c_{n+1}-2} 145671 \\
& w_{n+1}(4)=1(5674)^{3 c_{n+1}-2} 23745671 \\
& w_{n+1}(5)=15(7456)^{3 c_{n+1}-2} 7452371 \\
& w_{n+1}(6)=15(4567)^{3 c_{n+1}-2} 2367471 \\
& w_{n+1}(7)=12(5674)^{3 c_{n+1}-2} 3674571
\end{aligned}
$$

As before, one proves that the subdiagrams associated to the sets $\{1,2,3\}$ and $\{4,5,6,7\}$ define systems $(Y, S)$ and $(Z, R)$ respectively which are uniquely ergodic, and the extension of their unique probability measures are ergodic measures on $(X, T)$. Denote the ergodic measures on $(X, T)$ by $\widehat{\mu}$ and $\widehat{\nu}$. One also has that the diagram is clean and $I_{\widehat{\mu}}=\{1,2,3\}, I_{\widehat{\nu}}=\{4,5,6,7\}$. Thus, there is no other ergodic probability measure on $(X, T)$ aside from $\widehat{\mu}$ and $\widehat{\nu}$.

Now we sketch a proof that $\lambda=\exp (2 i \pi / 6)$ is a non continuous eigenvalue for $\widehat{\mu}$. Similarly one proves that $\lambda=\exp (2 i \pi / 8)$ is a non continuous eigenvalue for $\widehat{\nu}$. This last case is left to the reader.

First, a direct computation (one easily computes nine cases) serves to prove that for any $t_{1}, t_{2} \in\{1,2,3\}$, all, up to a bounded number of elements $s \in \bar{S}_{n}\left(t_{1}, t_{2}\right)$, are constant modulo 3. Denote $k\left(t_{1}, t_{2}\right)$ such a constant. Moreover, if $k\left(t_{1}, 1\right) \equiv_{3} c$ then $k\left(t_{1}, 2\right) \equiv_{3} c+1$ and $k\left(t_{1}, 3\right) \equiv_{3} c+2$; and if $k\left(1, t_{2}\right) \equiv_{3} c^{\prime}$ then $k\left(2, t_{2}\right) \equiv_{3} c^{\prime}+2$ 
and $k\left(3, t_{2}\right) \equiv_{3} c^{\prime}+1$. A precise inspection of values of $c$ and $c^{\prime}$ for all $t_{1}$ and $t_{2}$ allows to prove:

$$
k\left(t_{1}, t_{2}\right) \equiv_{3} k\left(t_{1}, t\right)+k\left(t, t_{2}\right) \text { for any } t \in\{1,2,3\} .
$$

This additive map is the one required by Corollary 4 . To finish the proof it is enough to produce a set $\mathcal{C}$ of full measure such that for any point $x \in \mathcal{C}$ one has $\bar{s}_{n}(x) \equiv_{3} k\left(\tau_{n}(x), \tau_{n+1}(x)\right)$ for all enough large $n \in \mathbb{N}$. As before, by considering for any $n \in \mathbb{N}$ the set

$$
\mathcal{C}_{n}=\left\{x \in X ; \bar{s}_{n}(x) \not \equiv_{3} k\left(\tau_{n}(x), \tau_{n+1}(x)\right)\right\}
$$

and using the fact that any $s \in \bar{S}_{n}\left(t_{1}, t_{2}\right)$ up to a bounded number of elements, say $L$, is constant modulo 3 , one gets that $\hat{\mu}\left(\mathcal{C}_{n}\right) \leq 3 L / q_{n+1}$. We finish the proof of the claim by the Borel-Cantelli Lemma, taking $\mathcal{C}=\liminf _{n \rightarrow \infty} \mathcal{C}_{n}^{c}$.

6.6. Example 6: Another (similar) big variation of the model example. Here we modify the previous example to provide a system with two ergodic measures and non continuous eigenvalues $\exp (2 i \pi / 6)$ and $\exp (2 i \pi / 4)$ respectively. This example shows that the first inequality of Corollary 7 (4) is an equality and the second is a strict inequality. For $t \in\{1, \ldots, 7\}$ and $n \geq 2$, consider the following choice of $w_{n+1}(t)$ and write $q_{n}=12 c_{n}+1$ :

$$
\begin{aligned}
& w_{n+1}(1)=(123)^{4 c_{n+1}-2} 1245671 \\
& w_{n+1}(2)=1(312)^{4 c_{n+1}-2} 345671 \\
& w_{n+1}(3)=1(123)^{4 c_{n+1}-2} 145671 \\
& w_{n+1}(4)=1(647465)^{2 c_{n+1}-1} 237461 \\
& w_{n+1}(5)=1(656574)^{2 c_{n+1}-1} 652361 \\
& w_{n+1}(6)=16(646575)^{2 c_{n+1}-1} 72361 \\
& w_{n+1}(7)=16(757564)^{2 c_{n+1}-1} 73261
\end{aligned}
$$

In this example the subdiagrams associated to $\{1,2,3\}$ and $\{4,5,6,7\}$ define systems $(Y, S)$ and $(Z, R)$ respectively which are uniquely ergodic, and the extensions of these ergodic measures, $\widehat{\mu}$ and $\widehat{\nu}$, are ergodic probability measures in $(X, T)$. As in the previous example there is no other ergodic probability measure on $(X, T)$. Furthermore, the diagram is clean, $I_{\widehat{\mu}}=\{1,2,3\}$ and $I_{\widehat{\nu}}=\{4,5,6,7\}$.

In relation to eigenvalues, doing similar computations as in the previous example one gets that $\exp (2 \pi i / 6)$ is a non continuous eigenvalue for $\widehat{\mu}$ and that $\exp (2 \pi i / 4)$ is a non continuous eigenvalue for $\widehat{\nu}$, while $\exp (2 \pi i / 8)$ is not.

\section{REFERENCES}

[BDM05] Xavier Bressaud, Fabien Durand, and Alejandro Maass, Necessary and sufficient conditions to be an eigenvalue for linearly recurrent dynamical Cantor systems, J. London Math. Soc. 72 (2005), 799-816.

[BDM10] - On the eigenvalues of finite rank Bratteli-Vershik dynamical systems, Ergod. Th. \& Dynam. Sys. 30 (2010), 639-664.

[BKMS13] S. Bezuglyi, J. Kwiatkowski, K. Medynets, and B. Solomyak, Finite rank Bratteli diagrams: structure of invariant measures, Trans. Amer. Math. Soc. 365 (2013), $2637-2679$.

[CDHM03] María Isabel Cortez, Fabien Durand, Bernard Host, and Alejandro Maass, Continuous and measurable eigenfunctions of linearly recurrent dynamical Cantor systems, J. London Math Soc. 67 (2003), 790-804. 
[Dek78] Frederik Michel Dekking, The spectrum of dynamical systems arising from substitutions of constant length, Z. Wahrscheinlichkeitstheorie und Verw. Gebiete 41 (1977/78), 221-239.

[DHS99] Fabien Durand, Bernard Host, and Christian Skau, Substitutional dynamical systems, Bratteli diagrams and dimension groups, Ergod. Th. \& Dynam. Sys. 19 (1999), 953993.

[DL96] Tomasz Downarowicz and Yves Lacroix, A non-regular Toeplitz flow with preset pure point spectrum, Studia Math. 120 (1996), 235-246.

[DM08] Tomasz Downarowicz and Alejandro Maass, Finite-rank Bratteli-Vershik diagrams are expansive, Ergod. Th. \& Dynam. Sys. 28 (2008), 739-747.

[Dur00] Fabien Durand, Linearly recurrent subshifts have a finite number of non-periodic subshift factors, Ergod. Th. \& Dynam. Sys. 20 (2000), 1061-1078.

[Dur03] Corrigendum and addendum to 'Linearly recurrent subshifts have a finite number of non-periodic subshift factors', Ergod. Th. \& Dynam. Sys. 23 (2003), 663669.

[Dur10] Fabien Durand, Combinatorics on Bratteli diagrams and dynamical systems, Combinatorics, automata and number theory, Encyclopedia Math. Appl., vol. 135, Cambridge Univ. Press, Cambridge, 2010, pp. 324-372.

[GJ00] Richard Gjerde and Ørjan Johansen, Bratteli-Vershik models for Cantor minimal systems: applications to Toeplitz flows, Ergod. Th. \& Dynam. Sys. 20 (2000), 16871710 .

[Hos86] Bernard Host, Valeurs propres des systèmes dynamiques définis par des substitutions de longueur variable, Ergod. Th. \& Dynam. Sys. 6 (1986), 529-540.

[HPS92] Richard Herman, Ian Putnam, and Christian Skau, Ordered Bratteli diagrams, dimension groups and topological dynamics, Int. J. Math. 3 (1992), 827-864.

[Iwa96] Anzelm Iwanik, Toeplitz flows with pure point spectrum, Studia Math. 118 (1996), $27-35$.

[JK69] Konrad Jacobs and Michael Keane, 0-1-sequences of Toeplitz type, Z. Wahrscheinlichkeitstheorie und Verw. Gebiete 13 (1969), 123-131.

[Wil84] Susan Williams, Toeplitz minimal flows which are not uniquely ergodic, Z. Wahrsch. Verw. Gebiete 67 (1984), 95-107.

Laboratoire Amiénois de Mathématiques Fondamentales et Appliquées, CNRS-UMR 7352, Université de Picardie Jules Verne, 33 rue Saint Leu, 80039 Amiens cedex 1, France.

E-mail address: fabien.durand@u-picardie.fr

Departamento de Ingeniería Matemática, Universidad de Chile, Avenida Blanco EncalADA 2120, Santiago, Chile.

E-mail address: afrank@dim.uchile.cl

Departamento de Ingeniería Matemática and Centro de Modelamiento Matemático, CNRS-UMi 2807, Universidad de Chile, Avenida Blanco Encalada 2120, Santiago, Chile.

E-mail address: amaass@dim.uchile.cl 